\title{
Imaginary-time formulation of steady-state nonequilibrium in quantum dot models
}

\author{
J. E. Han \\ Department of Physics, State University of New York at Buffalo, Buffalo, NY 14260, USA
}

(Dated: June 10, 2018)

\begin{abstract}
We examine the recently proposed imaginary-time formulation for strongly correlated steady-state nonequilibrium for its range of validity and discuss significant improvements in the analytic continuation of the Matsubara voltage as well as the fermionic Matsubara frequency. The discretization error in the conventional Hirsch-Fye algorithm has been compensated in the Fourier transformation with reliable small frequency behavior of self-energy. Here we give detailed discussions for generalized spectral representation ansatz by including high order vertex corrections and its numerical analytic continuation procedures. The differential conductance calculations agree accurately with existing data from other nonequilibrium transport theories. It is verified that, at finite source-drain voltage, the Kondo resonance is destroyed at bias comparable to the Kondo temperature. Calculated coefficients in the scaling relation of the zero bias anomaly fall within the range of experimental estimates.
\end{abstract}

PACS numbers: 73.63.Kv, 72.10.Bg, 72.10.Di

Progress in nanoscale fabrication techniques has recently generated a great deal of interest in electron transport out of equilibrium. Well-controlled quantum dot (QD) structures in semiconductor devices have enabled thorough investigation of quantum many-body effects in confined geometry. One of the established phenomena is the zero bias anomaly (ZBA) due to the Kondo effect in Coulomb blockade devices $\underline{1-3}$, where the scaling behavior of nonlinear conductance has been extensively verified. Recently, more complex quantum dot systems such as molecular nano-junctions ${ }^{4}$ and multi-channel Kondo $\operatorname{dots}^{\frac{5}{5}}$ with intricate device design have fueled intense research for electron transport mechanism in strongly correlated regime. Nanoscale single-electron devices hold great promise not only in applications for quantum devices but also in development of general quantum manybody theory out of equilibrium.

In the past few years, the strong correlation community has embraced the challenge of developing quantum manybody formulations out of equilibrium, which has lead to significant advances at various levels of theories. Unlike equilibrium quantum many-body theory where the analytic and numerical theories play complementary roles, the nonequilibrium theory has only recently had fullfledged numerical tools which could support or disprove the diagrammatic approximations, known as Keldysh technique $e^{6.7}$. So far, numerous algorithms have been proposed. However, most of the theories have yet to be fully established to have reliable predictive power in a wide range of strongly correlated transport.

The main focus of the theoretical efforts has been the description of the transient behavior toward a nonequilibrium steady-state or the properties of an established steady-state. Here the steady-state concerns the dc current-carrying state driven by a timeindependent bias in quantum dot geometry, as sketched in Fig. 1. Quantum simulations of real-time behavior of nonequilibrium steady-state have been performed using time-dependent numerical renormalization (a)
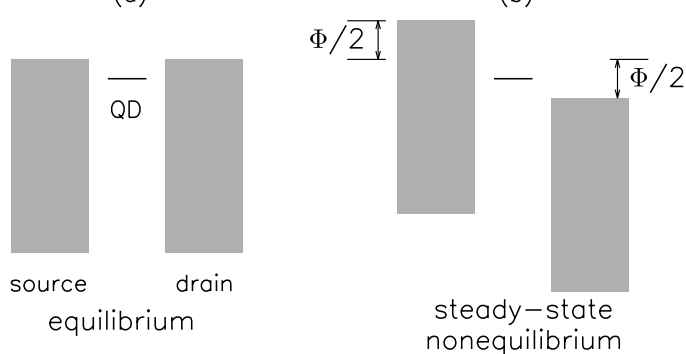

FIG. 1: (a) Source and drain reservoirs for electron and a quantum dot level. Chemical potentials of the reservoirs are the same in equilibrium. (b) Voltage bias $\Phi$ in steady-state nonequilibrium divided between chemical potentials of the reservoirs. The quantum dot energy level can be arbitrarily positioned with respect to the chemical potentials.

group (tNRG ${ }^{8}, 9$ ), time-dependent density-matrix $\mathrm{RG}$ (tDMRG 10,11$)$, perturbative RG (PRG $\left.{ }^{12,13}\right)$, functional renormalization group ( $\mathrm{fRG}^{14,15}$ ), iterative summation of real-time path-integral method (ISPI ${ }^{16,17}$ ), diagrammatic Monte Carlo $\frac{18,19}{20}$. Analytic methods of nonequilibrium Bethe ansatz ${ }^{20}$ and perturbative steady-state expansion 21 have been developed.

The imaginary-time formulation for steady-state nonequilibrium proposed by Han and Heary ${ }^{22}$ takes quite a different approach from the above real-time techniques. Main motivation has been to extend the equilibrium quantum many-body theory within a similar mathematical framework of the imaginary-time formalism and therefore to easily adopt existing equilibrium numerical techniques for complex quantum interactions such as molecular $\operatorname{dots}^{23}$. The method combines the equilibrium many-body theory and steady-state nonequilibrium quantum statistics by extending the chemical potentials into complex variables, the Matsubara voltage. It has 
been shown that, upon analytic continuation of complex chemical potential back to physical chemical potential the theory recovers the nonequilibrium dynamics, and the imaginary-time Green's functions map to real-time Green's functions.

In this review, we show how the previously introduced spectral ansatz can be extended to include vertex corrections and give comprehensive discussions detailing the justification and the range of validity of the analytic continuation. We further enhance the computational method by modifying the conventional Hirsch-Fye ${ }^{24}$ quantum Monte Carlo (QMC) algorithm by compensating the discretization error and obtain electron self-energy with much improved reliability. These improvements lead to reliable differential conductance curves in accurate agreement with other existing methods. We confirm that the Kondo resonance is destroyed at the bias of Kondo scale and the resulting scaling behavior of conductance is consistent with experiments.

The paper is organized as follows. In the following section [1 we define the imaginary-time Hamiltonian for nonequilibrium. We then show the equivalence of imaginary-time and real-time Green's functions in the perturbation expansion with arbitrary interaction through the analytic continuation. We give examples of different quantum dot geometry where this formalism can be extended. In the following section III we briefly introduce the QMC procedure and propose an algorithm which fixes discretization errors in the nonequilibrium QMC. In section III we extend the previously introduced analytic continuation ansatz via Padé approximants and describe detailed numerical procedures to fit spectral functions. We make a direct comparison of conductance in the small to intermediate interaction regimes with other methods. In section IV computational results are presented and compare them with existing theories and experiments. In the appendix, analytic structure of high order corrections to self-energy is discussed.

\section{IMAGINARY-TIME FORMALISM}

We start the discussion of nonequilibrium quantum theory with the understanding that the one of the fundamental problems is that there are two different operators governing the quantum statistics and the time-evolution. In equilibrium, the time-evolution is given by $e^{-i t H}$ while the Boltzmann factor $e^{-\beta(H-\mu N)}$ provides the quantum statistics with the chemical potential $\mu$ and the number operator $N$. With a number-conserving Hamiltonian, $[H, N]=0$, the Boltzmann factor and the time-evolution operator commute. For this reason, the chemical potential $\mu$ is often set as the reference energy $(\mu=0)$ without losing generality. However in nonequilibrium of quantum dot systems, bias voltage creates multiple electronic chemical potentials in the source and drain reservoirs. Once the tunneling between the quantum dot and the reservoirs is allowed and many-body inter- actions are turned on, the reservoir states mix with the time-evolution. Hence, the task of finding commutable nonequilibrium density matrix and the time-evolution operators becomes a challenging task.

From the 1960s and 1970s, efforts have been made to formulate a Gibbsian statistical mechanics in steadystate nonequilibrium using the Liouville operator formalism 25 . In 1993, Hershfield 26 has revisited the problem in the context of the mesoscopic transport and has provided a formal proof in a compact form of a density matrix for nonequilibrium steady-state. The idea consists of decomposing the full Hamiltonian in terms of the formal solution of scattering states of Lippman-Schwinger equation ${ }^{27}$, and applying different chemical potentials to scattering states derived from each reservoirs. With the scattering state operator $\psi_{\alpha k \sigma}^{\dagger}$ with the continuum index $k$, reservoir index $\alpha=L, R$ (or,+- , respectively) and the spin index $\sigma$, the nonequilibrium density matrix operator at the voltage bias $\Phi$ is written as

$$
\hat{\rho}=e^{-\beta(\hat{H}-\Phi \hat{Y})},
$$

with the operator $\hat{Y}$

$$
\hat{Y}=\frac{1}{2} \sum_{k \sigma}\left(\psi_{L k \sigma}^{\dagger} \psi_{L k \sigma}-\psi_{R k \sigma}^{\dagger} \psi_{R k \sigma}\right) .
$$

However, despite its conceptual breakthroughs, Hershfield's idea has not found practical implementations since fully interacting scattering states cannot be known a priori. The method has been adapted only in the noninteracting models and in perturbative limits 28,29 .

To overcome such difficulties, Han and Heary have proposed an imaginary-time formalism which constructs a formal perturbation expansion from a non-interacting nonequilibrium steady-state. The main advantage of the method over the original Hershfield's idea is that there is no need to construct the Hershfield's $\hat{Y}$-operator in the interacting limit. Many-body interactions are considered rigorously in a perturbation series at arbitrary order built on the non-interacting nonequilibrium density matrix $e^{-\beta\left(H_{0}-\Phi Y_{0}\right)}$ with the $\hat{Y}_{0}$-operator computed without many-body interactions. To compensate the discrepancy between the time-evolution and the Boltzmann factor, we introduce a mathematical trick of the Matsubara voltage.

Electron tunneling of single quantum dot connected to source and drain reservoirs is modeled by

$$
\begin{aligned}
\hat{H}_{0} & =\hat{H}_{0 c}+\hat{H}_{0 d}+\hat{H}_{0 t} \\
\hat{H}_{0 c} & =\sum_{\alpha k \sigma} \epsilon_{\alpha k} c_{\alpha k \sigma}^{\dagger} c_{\alpha k \sigma} \\
\hat{H}_{0 d} & =\epsilon_{d} \sum_{\sigma} d_{\sigma}^{\dagger} d_{\sigma} \\
\hat{H}_{0 t} & =-\sum_{\alpha k \sigma} \frac{t_{\alpha}}{\sqrt{\Omega}}\left(d_{\sigma}^{\dagger} c_{\alpha k \sigma}+\text { h.c. }\right),
\end{aligned}
$$

with $\hat{H}_{0 c}, \hat{H}_{0 d}, \hat{H}_{0 t}$ for decoupled reservoirs, quantum dot states, and QD-reservoir tunneling, respectively. $c_{\alpha k \sigma}^{\dagger}$ are 
the fermion operators for continuum state, $d_{\sigma}^{\dagger}$ quantum dot orbital operator, $t_{\alpha}$ the tunneling parameter and $\Omega$ the volume of the reservoirs. Then the non-interacting scattering state can be readily written down as $\frac{28,29}{}$

$$
\begin{aligned}
\psi_{0, \alpha k \sigma}^{\dagger}= & c_{\alpha k \sigma}^{\dagger}-\frac{t_{\alpha}}{\sqrt{\Omega}} g_{d}^{0}\left(\epsilon_{\alpha k}\right) d_{\sigma}^{\dagger} \\
& +\sum_{\alpha^{\prime} k^{\prime}} \frac{t_{\alpha} t_{\alpha^{\prime}}}{\Omega} \frac{g_{d}^{0}\left(\epsilon_{\alpha k}\right)}{\epsilon_{\alpha k}-\epsilon_{\alpha^{\prime} k^{\prime}}+i \eta} c_{\alpha^{\prime} k^{\prime} \sigma}^{\dagger}
\end{aligned}
$$

with the non-interacting retarded Green's function $g^{0}(\omega)=\left[\omega-\epsilon_{d}+i \Gamma\right]^{-1} . \quad \Gamma$ is the line-broadening of the quantum dot $\Gamma=\Gamma_{L}+\Gamma_{R}=\pi\left(t_{L}^{2}+t_{R}^{2}\right) N(0)$ with the density of states of the reservoirs $N(0)$.

Even in the non-interacting limit, the problem of combining the density matrix and the time-evolution operator remains. To resolve the issue, we extend the chemical potential into complex numbers such that the new chemical potential does not alter the quantum statistics while the time-evolution rate along the imaginary-time can be shifted. We introduce an effective non-interacting Hamiltonian $\hat{K}_{0}$,

$$
\hat{K}_{0}=\hat{H}_{0}+\left(i \varphi_{m}-\Phi\right) \hat{Y}_{0},
$$

with the Matsubara voltage,

$$
i \varphi_{m}=i \frac{4 \pi m}{\beta} \text { for any integer } m .
$$

With the addition of the Matsubara voltage, the density matrix given by $\hat{K}_{0}$ becomes $e^{-\beta K_{0}}=e^{-\beta\left[H_{0}+\left(i \varphi_{m}-\Phi\right) Y_{0}\right]}$. Since $\left[H_{0}, Y_{0}\right]=0$, the operator can be written as $e^{-\beta\left(H_{0}-\Phi Y_{0}\right)} \cdot e^{-i \beta \varphi_{m} Y_{0}}$. With respect to the Fock basis constructed from the non-interacting operators $\psi_{0, \alpha k \sigma}^{\dagger}$, $H_{0}$ and $Y_{0}$ are diagonal and the eigenvalues of $Y_{0}$ are halfor full-integers. Therefore, we have an operator identity $e^{-i \beta \varphi_{m} Y_{0}}=1$ and

$$
e^{-\beta K_{0}}=e^{-\beta\left(H_{0}-\Phi Y_{0}\right)}=\hat{\rho}_{0},
$$

independent of $i \varphi_{m}$. This effective Hamiltonian amounts to a electron system with the statistics given by $\hat{\rho}_{0}$ and the time-evolution is controlled at an independent phase velocity shifted by the pumping frequency of $i \varphi_{m}$. This extra complex term is only applied to the imaginary-time action and should not be interpreted as a physical damping term in real-time formalism.

Given the non-interacting Hamiltonian, Eq. (8), we introduce the interacting Hamiltonian $\hat{K}$, parametrized by $i \varphi_{m}-\Phi$, with the many-body interaction $\hat{V}$ as

$$
\hat{K}=\hat{K}_{0}+\hat{V}=\hat{H}_{0}+\left(i \varphi_{m}-\Phi\right) \hat{Y}_{0}+\hat{V},
$$

and treat this as an effective Hamiltonian to the equilibrium imaginary-time theory. In the following section, we will show that, upon the analytic continuation $i \varphi_{m} \rightarrow \Phi$ the thermal Green's functions map to nonequilibrium real-time Green's functions.
The equivalence of the imaginary-time theory and the real-time Keldysh formalism can be best shown in explicit perturbative expansions. The real-time retarded Green's function for the quantum dot is defined as

$$
\begin{aligned}
G^{r e t}(t) & =-i \theta(t)\left\langle\left\{d_{H}(t), d_{H}^{\dagger}(0)\right\}\right\rangle \\
& =\theta(t)\left[G^{>}(t)-G^{<}(t)\right],
\end{aligned}
$$

with the lesser and greater Green's functions defined as

$$
\begin{aligned}
& G^{>}(t)=-i\left\langle d_{H}(t) d_{H}^{\dagger}(0)\right\rangle \\
& G^{<}(t)=i\left\langle d_{H}^{\dagger}(0) d_{H}(t)\right\rangle .
\end{aligned}
$$

The subscript $H$ refers to the evolution in the Heisenberg picture with the full Hamiltonian, $d^{\dagger}(t)=e^{i t H} d^{\dagger} e^{-i t H}$. $\theta(t)$ is the step-function.

Usually an interaction picture is defined with a timedependent many-body interaction $\hat{V}$ turned on adiabatically from the infinite past $(t=T$ as $T \rightarrow-\infty$, see Fig. 21). The Green's functions can be defined in the Keldysh contour as

$$
G^{>}(t)=-i Z_{0, n e q}^{-1} \operatorname{Tr}\left[\hat{\rho}_{0} \mathcal{T}_{K} e^{-i \int_{K} V_{I}\left(t^{\prime}\right) d t^{\prime}} d(t) d^{\dagger}(0)\right] .
$$

The real-time evolution is given in the interaction picture as $d^{\dagger}(t)=e^{i t H_{0}} d^{\dagger} e^{-i t H_{0}}$ and $\hat{V}_{I}(t) e^{i t H_{0}} \hat{V} e^{-i t H_{0}}$. Time variables are defined on the Keldysh contour, denoted as $K$. The nonequilibrium partition function is defined as $Z_{0, \text { neq }}=\operatorname{Tr} \hat{\rho}_{0}$.

Here, to avoid explicit time-dependence to the interaction $\hat{V}$, we use Gell-Mann and Goldberger' ${ }^{30}$ construction of steady-state when the limit $T \rightarrow-\infty$ is taken. Their formalism uses full-strength interaction instead of adiabatic interaction with the turn-on time $T$ integrated from the remote past to the present. For example, a scattering state operator defined as

$$
\psi_{k}^{\dagger}=\eta \int_{-\infty}^{0} d T e^{\eta T} e^{-i \mathcal{L} T} e^{i \mathcal{L}_{0 c} T} c_{k}^{\dagger},
$$

satisfies the Lippman-Schwinger equation

$$
\psi_{k}^{\dagger}=c_{k}^{\dagger}+\frac{1}{\epsilon_{k}-\mathcal{L}+i \eta}\left[\hat{V}+\hat{H}_{0 t}, c_{k}^{\dagger}\right],
$$

with the tunneling part of the non-interacting Hamiltonian $\hat{H}_{0 t}$. Here the infinitesimal $\eta$ determines the direction of the time propagation. The Liouville operators are defined as $\mathcal{L} \hat{A}=[\hat{H}, \hat{A}], \mathcal{L}_{0 c} \hat{A}=\left[\hat{H}_{0 c}, \hat{A}\right]$, and $e^{-i \mathcal{L} T} \hat{A}=e^{-i \hat{H} T} \hat{A} e^{i \hat{H} T}$, etc. Here the adiabatic limit $T \rightarrow-\infty$ is replaced by an integral via $\eta \int_{-\infty}^{0} d T e^{\eta T}$ where the start time $T$ of interaction is averaged over the whole range $(-\infty, 0]$. This averaging effectively cancels out the transient phase of each scattered wave which has propagated with the interaction turned on at time $T$.

The greater Green's function $G^{>}(t)$ can be represented as Fig. 2 with a $d$-electron created at time 0 on the upper contour and an electron destroyed at time $t$ on the 


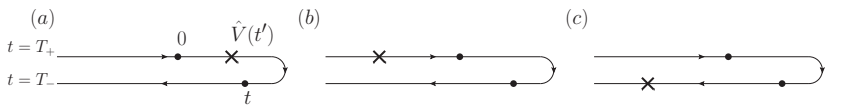

FIG. 2: (a) Keldysh contour for greater Green's function with the first order scattering (marked by $\mathrm{X}$ ) happening between time $t=0$ and $t$ [corresponding to the case (i) considered in the text] (b) Time ordering of case (ii). (c) Case (iii).

lower contour. Then, the first order perturbation correction from Eq. (16) can be decomposed into three different time-ordered terms according to the time of interaction $t^{\prime}$ as (i) $0_{+}<t^{\prime}<t_{-}$(ii) $T_{+}<t^{\prime}<0_{+}$(iii) $t_{-}<t^{\prime}<T_{-}$, as shown in Figs. 2(a)-(c), respectively. The contribution for (i) can be expressed as (with $Z_{0, n e q}^{-1}$ omitted for brevity)

$$
\begin{aligned}
& -i \int_{0}^{t}\left\langle e^{-i(T-t) H_{0}} d e^{-i\left(t-t^{\prime}\right) H_{0}} \hat{V} e^{-i t^{\prime} H_{0}} d^{\dagger} e^{i T H_{0}}\right\rangle_{0} d t^{\prime} \\
= & -i \int_{0}^{t}\left\langle e^{i t H_{0}} d e^{-i\left(t-t^{\prime}\right) H_{0}} \hat{V} e^{-i t^{\prime} H_{0}} d^{\dagger}\right\rangle_{0} d t^{\prime} \\
= & -i \int_{0}^{t} \sum_{n m k} \hat{\rho}_{0, n} e^{i t\left(E_{n}-E_{m}\right)} d_{n m} e^{i t^{\prime}\left(E_{m}-E_{k}\right)} \hat{V}_{m k} d_{k n}^{\dagger} d t^{\prime} \\
= & \left.-\sum_{n m k} \hat{\rho}_{0, n}\left[e^{i t\left(E_{n}-E_{m}\right)}-e^{i t\left(E_{n}-E_{k}\right)}\right] \frac{d_{n m} \hat{V}_{m k} d_{k n}^{\dagger}}{E_{m}-E_{k}} 19\right)
\end{aligned}
$$

where the states denoted by $n, m, k$ are Fock states constructed from the non-interacting scattering states. $\langle\cdots\rangle_{0}$ is defined as $\operatorname{Tr}\left[\hat{\rho}_{0} \cdots\right]$. Note that zero of the energy denominator $E_{m}-E_{k}$ does not lead to a singularity since $e^{i t\left(E_{n}-E_{m}\right)}-e^{i t\left(E_{n}-E_{k}\right)}=0$.

For the perturbation occurring in the interval extending to the infinity on the upper time contour [Fig. 2(b)], the integral for (ii) becomes

$$
\begin{aligned}
& -i \eta \int_{-\infty}^{0} d T e^{\eta T} \int_{T}^{0} d t^{\prime}\left\langle e^{i t H_{0}} d e^{-i t H_{0}} d^{\dagger} e^{i t^{\prime} H_{0}} \hat{V} e^{-i t^{\prime} H_{0}}\right\rangle_{0} \\
& =-\sum_{n m k} \hat{\rho}_{0, n} e^{i t \Delta E_{n m}} \frac{d_{n m} d_{m k}^{\dagger} \hat{V}_{k n}}{\Delta E_{k n}-i \eta}
\end{aligned}
$$

with $\Delta E_{n m}=E_{n}-E_{m}$. Similarly for the case (iii) with the interaction on the lower contour, we have

$$
\begin{aligned}
& -i \eta \int_{-\infty}^{0} d T e^{\eta T} \int_{t}^{T} d t^{\prime}\left\langle e^{i t^{\prime} H_{0}} \hat{V} e^{-i\left(t^{\prime}-t\right) H_{0}} d e^{-i t H_{0}} d^{\dagger}\right\rangle_{0} \\
& =\sum_{n m k} \hat{\rho}_{0, n} e^{i t \Delta E_{n k}} \frac{\hat{V}_{n m} d_{m k} d_{k n}^{\dagger}}{\Delta E_{n m}-i \eta}
\end{aligned}
$$

By denoting the time ordering on the Keldysh contour as $(a b)_{K}$ for an event $a$ following $b$, the Eqs. 119. 21) can be represented by a cyclic permutation of $\left\{\left(d V d^{\dagger}\right)_{K},\left(d d^{\dagger} V\right)_{K},\left(V d d^{\dagger}\right)_{K}\right\}$, respectively. Rearranging the indices in Eqs. (20]21), the contributions from (ii) and (iii) combine to

$$
\sum_{n m k}\left[\hat{\rho}_{0, n} e^{i t \Delta E_{n k}}-\hat{\rho}_{0, m} e^{i t \Delta E_{m k}}\right] \frac{\hat{V}_{n m} d_{m k} d_{k n}^{\dagger}}{\Delta E_{n m}-i \eta} .
$$

Finally the first-order perturbation to the greater Green's function becomes

$$
\begin{aligned}
& -\sum_{n m k} \hat{\rho}_{0, n}\left[e^{i t \Delta E_{n m}}-e^{i t \Delta E_{n k}}\right] \frac{d_{n m} \hat{V}_{m k} d_{k n}^{\dagger}}{\Delta E_{m k}} \\
& +\sum_{n m k}\left[\hat{\rho}_{0, n} e^{i t \Delta E_{n k}}-\hat{\rho}_{0, m} e^{i t \Delta E_{m k}}\right] \frac{\hat{V}_{n m} d_{m k} d_{k n}^{\dagger}}{\Delta E_{n m}-i \eta}(23)
\end{aligned}
$$

We perform the same perturbation theory to the thermal Green's function defined with the imaginary-time under the Hamiltonian $\hat{K}$, Eq. (11), as

$$
\mathcal{G}(t)=-\left\langle\mathcal{T}_{\tau} d_{K}(\tau) d_{K}^{\dagger}(0)\right\rangle
$$

with the time-evolution given as $d_{K}(\tau)=e^{\tau K} d e^{-\tau K}$. Using the interaction picture, the Green's function is expanded in a perturbation series as

$$
\mathcal{G}(t)=-Z_{0, n e q}^{-1} \operatorname{Tr}\left[\hat{\rho}_{0} \mathcal{T}_{\tau} e^{-\int_{0}^{\beta} V_{I}\left(\tau^{\prime}\right) d \tau^{\prime}} d(\tau) d^{\dagger}(0)\right],
$$

where the time-evolution in the interaction picture is given as $d(\tau)=e^{\tau K_{0}} d e^{-\tau K_{0}}$. For $\tau>0$, the first perturbation contribution due to the scattering of $\hat{V}$ at $\tau^{\prime}$ can be grouped into two cases; (i) $0<\tau^{\prime}<\tau$ and (ii) $\tau<\tau^{\prime}<\beta$.

The contribution from (i) $0<\tau^{\prime}<\tau$ is

$$
\begin{aligned}
& -\int_{0}^{\tau} \operatorname{Tr}\left[e^{-(\beta-\tau) K_{0}} d e^{-\left(\tau-\tau^{\prime}\right) K_{0}} \hat{V} e^{-\tau^{\prime} K_{0}} d^{\dagger}\right] d \tau^{\prime} \\
= & \sum_{n m k} \rho_{0, n}\left[e^{\tau \Delta K_{n m}}-e^{\tau \Delta K_{n k}}\right] \frac{d_{n m} \hat{V}_{m k} d_{k n}^{\dagger}}{\Delta K_{m k}}
\end{aligned}
$$

with the eigenvalues $K_{n}$ of $\hat{K}_{0}$ and $\Delta K_{n m}=K_{n}-K_{m}$. Here we have used the key relation Eq. (10), $e^{-\beta \hat{K}_{0}}=\hat{\rho}_{0}$. If the analytic continuation $i \varphi_{m} \rightarrow \Phi$ is formally carried out followed by $\tau \rightarrow i t, K_{n} \rightarrow E_{n}$ and the above integral for $0<\tau^{\prime}<\tau$ becomes identical to the real-time Green's function Eq. (19) for $0_{+}<t^{\prime}<t_{-}$. Now, for the case of (ii) $\tau<\tau^{\prime}<\beta$, the integral becomes

$$
\begin{aligned}
& -\int_{\tau}^{\beta} \operatorname{Tr}\left[e^{-\left(\beta-\tau^{\prime}\right) K_{0}} \hat{V} e^{-\left(\tau^{\prime}-\tau\right) K_{0}} d e^{-\tau K_{0}} d^{\dagger}\right] d \tau^{\prime} \\
= & \sum_{n m k}\left[\rho_{0, n} e^{\tau \Delta K_{n k}}-\rho_{0, m} e^{\tau \Delta K_{m k}}\right] \frac{\hat{V}_{n m} d_{m k} d_{k n}^{\dagger}}{\Delta K_{n m}},(27)
\end{aligned}
$$

which transforms to the real-time result, Eq. (21), with the analytic continuation except for the adiabatic factor in.

We discuss the subtlety of the adiabatic factor $i \eta$, the presence of which is only relevant for the energy shell of 
(a)

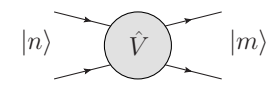

(b)

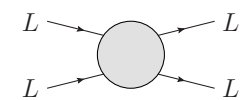

(c)
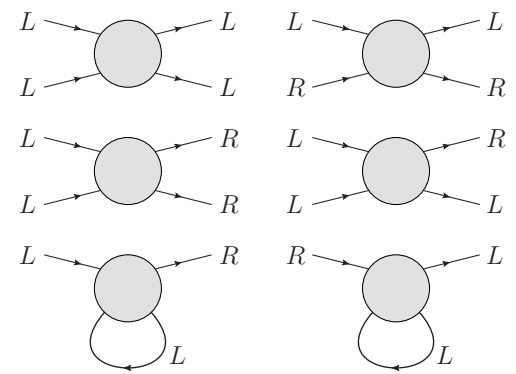

$\longrightarrow$

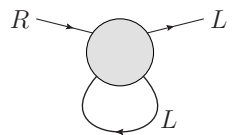

FIG. 3: (a) Interaction $\hat{V}$ mapping a state $|n\rangle$ to $|m\rangle$. (b) Initial and final states without changes in the $Y_{0}$-number of scattering states. (c) Initial and final states with different $Y_{0}$-numbers. (d) Contraction with the $d$-creation and annihilation operators. Each line represents the contraction of scattering state basis $\left\langle\psi_{\alpha k \sigma}^{\dagger} \psi_{\alpha k \sigma}\right\rangle_{0}$ and contributes the factor $t_{\alpha}^{2}\left|g\left(\epsilon_{\alpha k}\right)\right|^{2}$. Two diagrams are with $|n\rangle$ and $|m\rangle$ states interchanged.

$E_{n}=E_{m}$ in Eq. (22),

$$
i \pi \sum_{n m k}\left[\hat{\rho}_{0, n}-\hat{\rho}_{0, m}\right] e^{i t \Delta E_{n k}} \hat{V}_{n m} d_{m k} d_{k n}^{\dagger} \delta\left(E_{n}-E_{m}\right) .
$$

In equilibrium, $\hat{\rho}_{0}$ is only given by energy and the above expression is zero since $\rho_{0, n}-\rho_{0, m}=0$. To extend the argument to nonequilibrium, we take an explicit example of one-quantum dot with a two-body interaction such as the on-site Coulomb interaction $\hat{V}=U n_{d \uparrow} n_{d \downarrow}$. In terms of the explicit scattering state operators Eq. (7),

$$
\begin{aligned}
d_{\sigma}^{\dagger}= & \sum_{\alpha k} \frac{t_{\alpha}}{\sqrt{\Omega}} g^{*}\left(\epsilon_{\alpha k}\right) \psi_{\alpha k \sigma}^{\dagger} \\
\hat{V}= & U \sum_{\{\alpha, k\}}\left(\frac{t_{\alpha_{1}} g^{*}\left(\epsilon_{1}\right)}{\sqrt{\Omega}} \psi_{\alpha 1 k 1 \uparrow}^{\dagger}\right)\left(\frac{t_{\alpha_{2}} g\left(\epsilon_{2}\right)}{\sqrt{\Omega}} \psi_{\alpha 2 k 2 \uparrow}\right) \\
& \times\left(\frac{t_{\alpha_{3}} g^{*}\left(\epsilon_{3}\right)}{\sqrt{\Omega}} \psi_{\alpha 3 k 3 \downarrow}^{\dagger}\right)\left(\frac{t_{\alpha_{4}} g\left(\epsilon_{4}\right)}{\sqrt{\Omega}} \psi_{\alpha 4 k 4 \downarrow}\right) .
\end{aligned}
$$

As depicted in the Fig. 3(b), if the incoming and outgoing states $|n\rangle$ and $|m\rangle$ conserve the eigenvalues for $\hat{Y}_{0}$ operator $\left(Y_{0}\right.$-number $)$, the factor $\left(\hat{\rho}_{0, n}-\hat{\rho}_{0, m}\right)$ is zero. Figure [3(c) shows the terms in $\hat{V}$ [Eq. (30)] which do not conserve the $Y_{0}$-number. However, when expectation values are taken between the states $|n\rangle$ and $|m\rangle$, it can be shown that there is a counter-term which leads to a cancellation. As demonstrated in Fig. 3(d), the connected legs represent the contraction $\left\langle\psi_{\alpha k \sigma}^{\dagger} \psi_{\alpha k \sigma}\right\rangle_{0}$ and contribute the factor $t_{\alpha}^{2}\left|g\left(\epsilon_{\alpha k}\right)\right|^{2}$ from Eqs. (7) and (30). If the source and drain reservoirs are given by the same continuum density of states, there is always a contribution with the same magnitude from when the states $|n\rangle$ and $|m\rangle$ are interchanged, therefore leading to the cancellation of the factor $\left(\hat{\rho}_{0, n}-\hat{\rho}_{0, m}\right)$. We emphasize that the above argument does not require assumptions for the
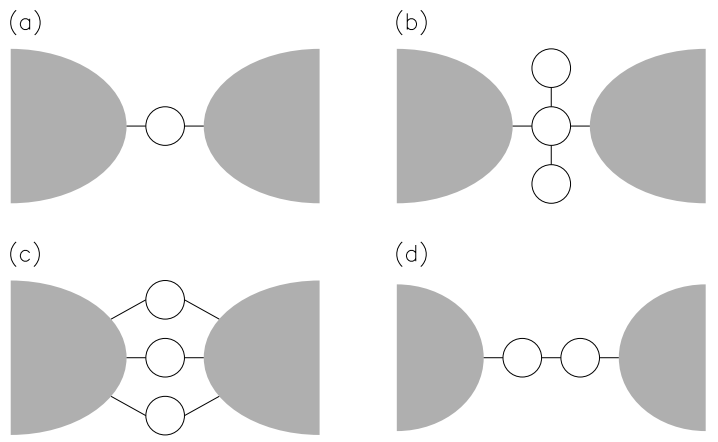

FIG. 4: Nonlinear Transport through (a) single QD, (b) sidecoupled QDs and (c) parallel QDs can be studied within the imaginary-time formalism. (d) Green's functions in serially coupled QDs may not be correctly continued to lesser Green's functions.

source-drain symmetry $t_{L}=t_{R}$ or the particle-hole symmetry. It also holds for different types of on-site interaction.

To summarize, the time-ordering of the realtime greater Green's function can be matched to the imaginary-time-ordering [denoted by $(\cdots)_{I}$ ] as $\left(d V d^{\dagger}\right)_{K} \leftrightarrow\left(d V d^{\dagger}\right)_{I}$ and $\left(V d d^{\dagger}\right)_{K}+\left(d d^{\dagger} V\right)_{K} \leftrightarrow$ $\left(V d d^{\dagger}\right)_{I}$. The higher order contributions can be checked in the similar manner to the first order. For example, in the second order, the time-orderings in the realtime theory can be matched as $\left(d V V d^{\dagger}\right)_{K} \leftrightarrow\left(d V V d^{\dagger}\right)_{I}$, $\left(V d V d^{\dagger}\right)_{K}+\left(d V d^{\dagger} V\right)_{K} \leftrightarrow\left(V d V d^{\dagger}\right)_{I}$, and $\left(V V d d^{\dagger}\right)_{K}+$ $\left(V d d^{\dagger} V\right)_{K}+\left(d d^{\dagger} V V\right)_{K} \leftrightarrow\left(V V d d^{\dagger}\right)_{I}$. Such topologically equivalent graphs between the imaginary-time and real-time expansions at each perturbation order are expected since the two theories are known to be equivalent in equilibrium.

The lesser Green's function can be shown to be equivalent to the imaginary-time Green's function $\mathcal{G}(\tau)$ for $\tau<0$. As shown previously ${ }^{22}$, if Fourier transformation is performed on the real- and imaginary-time Green's functions, the retarded Green's function $G^{\text {ret }}(\omega)$ can be obtained by analytically continuing the thermal Green's function $\mathcal{G}\left(i \omega_{n}\right)$ via $i \omega_{n} \leftrightarrow \omega+i \eta$.

So far, we discussed the analytic continuation in singleQD structures [Fig. 4(a)]. However, the formulation can be straightforwardly extended to much wider range of quantum dot structures such as the side-coupled QD and parallel-coupled QD systems as shown in Fig. 4(b-c). For serially coupled QD systems [Fig. 4(d)], the current imaginary-time formulation does not have an analytical continuation to real-time Green's functions and hence the formulation in this work cannot be applied.

\section{COMPUTATIONAL ALGORITHM}

We implement the imaginary-time formalism for a numerical nonequilibrium technique using quantum Monte 
Carlo method. We use the Hirsch-Fye (HF) algorithm ${ }^{24}$ which uses only the interacting orbitals (QD site) as the dynamic variable after integrating out the noninteracting reservoir electronic states. The QD Green's function is stochastically updated along the discretized imaginary-time lattice using the local update method by Blankenbecler et $\mathrm{al}^{32}$. Calculation of nonequilibrium interacting Green's function does not require any main modifications of the standard (equilibrium) QMC code.

\section{A. Non-interacting Green's function}

Independent sets of simulations are performed with different $i \varphi_{m}$ 's in Eq. (11) treated as fixed parameters to QMC. In the following calculations, $0 \leq m \leq 5$ have been used. Given $i \varphi_{m}$, the non-interacting QD Green's function can be easily derived as

$$
\begin{aligned}
\mathcal{G}^{0}\left(i \omega_{n}\right) & =\sum_{\alpha k}\left\langle d_{\sigma} \mid \psi_{\alpha k \sigma}\right\rangle \frac{1}{i \omega_{n}-K_{0, \alpha k}}\left\langle\psi_{\alpha k \sigma} \mid d_{\sigma}\right\rangle \\
& =\sum_{\alpha k} \frac{t_{\alpha}^{2}}{\Omega} \frac{\left|g\left(\epsilon_{\alpha k}\right)\right|^{2}}{i \omega_{n}-\epsilon_{\alpha k}-\alpha \frac{i \varphi_{m}-\Phi}{2}} \\
& =\sum_{\alpha} \frac{\Gamma_{\alpha} / \Gamma}{z_{n m}+i \Gamma \cdot \operatorname{Sign}\left(\operatorname{Im} z_{n m}\right)}
\end{aligned}
$$

with $z_{n m}=i \omega_{n}-\alpha \frac{i \varphi_{m}-\Phi}{2}$. If the analytic continuation of $i \varphi_{m} \rightarrow \Phi$ followed by $i \omega_{n} \rightarrow \omega+i \eta$ is performed, the thermal Green's function in the Matsubara frequency transforms to the real-time retarded Green's function,

$$
\sum_{\alpha} \frac{\Gamma_{\alpha} / \Gamma}{\omega+i \eta+i \Gamma}=\frac{1}{\omega+i \eta+i \Gamma} .
$$

Fourier transformation to the imaginary-time variable $(\tau>0)$ gives

$$
\begin{aligned}
\mathcal{G}^{0}(\tau) & =\frac{1}{\beta} \sum_{n} \mathcal{G}^{0}\left(i \omega_{n}\right) e^{-i \omega_{n} \tau} \\
& =-\sum_{\alpha k} \frac{t_{\alpha}^{2}}{\Omega}\left|g^{0}\left(\epsilon_{\alpha k}\right)\right|^{2} e^{-\tau\left[\epsilon_{\alpha k}+\alpha\left(i \varphi_{m}-\Phi\right) / 2\right]}\left[1-f_{\alpha}(\right.
\end{aligned}
$$

with the Fermi-Dirac function in the $\alpha$-reservoir, $f_{\alpha}(\epsilon)=$ $\left[1+e^{\beta(\epsilon-\alpha \Phi / 2)}\right]^{-1}$. This expression is later used as the input to the QMC calculation. If we perform the analytic continuation $i \varphi_{m} \rightarrow \Phi$ followed by $\tau \rightarrow i t$, the Green's function in the imaginary-time transforms to

$$
-\sum_{\alpha k} \frac{t_{\alpha}^{2}}{\Omega}\left|g^{0}\left(\epsilon_{\alpha k}\right)\right|^{2} e^{-i t \epsilon_{\alpha k}}\left[1-f_{\alpha}\left(\epsilon_{\alpha k}\right)\right],
$$

which is nothing but the non-interacting greater Green's function of $\mathrm{QD}$ orbital, $G^{>}(t)$, in the steady-state nonequilibrium. In this sense, the thermal Green's function before analytic continuations contains full information of the retarded, greater and lesser Green's functions in the real-time formulation.
In this work, we analytically continue the retarded (self-energy) functions instead of the lesser/greater Green's functions as functions of frequency since the retarded functions have simpler analytic structure of rational functions, as opposed to the exponential functions for the lesser/greater Green's functions as functions of time.

\section{B. Quantum Monte-Carlo method and Self-energy}

The QMC procedure follows the standard Hirsch-Fye algorithm with the initial Green's function Eq. (36) at fixed $i \varphi_{m}$. QMC method stochastically samples the fermionic phase space via auxiliary fields in the action introduced by the Hubbard-Stratonovich transformation ${ }^{24}$. The auxiliary fields are updated according to the effective Boltzmann factor ${ }^{32}$. The only modification to the HF algorithm is that the Monte Carlo (MC) Green's function $G\left(\tau, \tau^{\prime}\right)$ at an auxiliary field configuration is complex in contrast to the equilibrium calculations. Since $G\left(\tau, \tau^{\prime}\right)$ is complex, the ratio of Boltzmann factors for the new and old auxiliary field configurations is also complex in general. Therefore for any observable $\langle\hat{A}\rangle$ we compute the ensemble average as

$$
\langle\hat{A}\rangle=\frac{\sum_{n} f(n) A(n)}{\sum_{n} f(n)}=\frac{\sum_{n} e^{i \theta_{n}} A(n)|f(n)|}{\sum_{n} e^{i \theta_{n}}|f(n)|}=\frac{\left.\left\langle e^{i \theta} A\right\rangle\right\rangle}{\left\langle\left\langle e^{i \theta}\right\rangle\right\rangle},
$$

with the effective Boltzmann factor $f(n)$ for a auxiliary field configuration $n$ and its phase factor $e^{i \theta_{n}}=$ $f(n) /|f(n)|$. The ensemble average $\langle\langle\cdots\rangle$ is taken over the Markov chain of the Monte Carlo configurations chosen by the probability $|f(n)|$. In the calculations shown later, the average phase factor $\left|\left\langle\left\langle e^{i \theta}\right\rangle\right\rangle\right|$ remained close to one (typically $0.9-1.0$ ) and the statistics has been quite robust. We note that at $\Phi=0$ and $i \varphi_{m}=0$, the QMC calculation is completely identical to the equilibrium QMC method.

The QMC Green's function defined on a discrete imaginary-time mesh $\tau_{i}=i \Delta \tau(\Delta \tau=\beta / N, i=$ $0, \cdots, N-1)$ is updated by the QMC Dyson's equa$\operatorname{tion}^{24,32}$

$$
\mathbf{G}=\mathbf{G}_{0}+\left(\mathbf{G}_{0}-I\right)\left(e^{\mathbf{V}}-I\right) \mathbf{G},
$$

for the Green's function matrix defined as $\mathbf{G}_{i j}=$ $G\left(\tau_{i}, \tau_{j}\right)$. Here $\mathbf{V}$ represents the auxiliary-field coupling to electrons after the Hubbard-Stratonovich transformation $^{24,32}$ of many-body interaction. The diagonal component of the matrix $\mathbf{G}$ is chosen as the greater Green's function by ${ }^{33}$

$$
G_{i i}=G^{>}\left(\tau_{i}, \tau_{i}\right)=G\left(\tau_{i}+0^{+}, \tau_{i}\right) .
$$

This choice of zero-time Green's function introduces discretization error when the time variables are integrated in the above Dyson's equation or in other observables. In nonequilibrium calculations, the Green's function, Eq. (36), has an additional oscillation due to the $i \varphi_{m}$ 
dependence, and the systematic error of discretization becomes more significant. Spurious structures in the low frequency self-energy observed in $\operatorname{Ref}^{22}$ are attributed to the discretization error ${ }^{34}$, which can be confirmed in comparison with the continuous-time QMC 35,36 . In Figs. 5 (ab), the discretization error is compared for $\Delta \tau=1 / 5$ and $1 / 10$. The energy unit is the non-interacting broadening $\Gamma$. Although the self-energy at high frequency $\omega_{n}$ is well convergent, the low frequency $\Sigma\left(i \omega_{n}, i \varphi_{m}\right)$ shows discontinuous jumps at $\omega_{n} \approx 0$ as $\varphi_{m}$ increases. This kink becomes smoother as $\Delta \tau$ becomes small. Without the correction, the analytic continuation misinterprets the kink in the self-energy due to incoherent spectra and exaggerated the destruction of Kondo resonance at finite bias 22 .

By adopting a similar trick for Fourier transformation considered in the continuous-time QMC ${ }^{35.36}$, the Green's functions in the discrete-time QMC has been measured as follows. When involved in a time-integral, we use the non-interacting Green's function matrix $\tilde{\mathbf{G}}_{0}$ with the diagonal elements augmented by $\tilde{\mathbf{G}}_{0, i i}=$ $\frac{1}{2}\left[G_{0}\left(\tau_{i}+0^{+}, \tau_{i}\right)+G_{0}\left(\tau_{i}-0^{+}, \tau_{i}\right)\right]=G_{0}\left(\tau_{i}+0^{+}, \tau_{i}\right)-\frac{1}{2}$, i.e. $\tilde{\mathbf{G}}_{0}=\mathbf{G}_{0}-\frac{1}{2} I$. Then the Dyson's equation can be rewritten as

$$
\mathbf{G}=\mathbf{G}_{0}+\tilde{\mathbf{G}}_{0} \mathbf{S} \tilde{\mathbf{G}}_{0}+\frac{1}{2}\left(\mathbf{S} \tilde{\mathbf{G}}_{0}-\tilde{\mathbf{G}}_{0} \mathbf{S}\right),
$$

with the $S$-matrix defined as

$$
\mathbf{S}=\left(e^{\mathbf{V}}-I\right) \mathbf{G G}_{0}^{-1} .
$$

Through Monte Carlo updates we measure the Fourier transformed $S$-matrix as

$$
S\left(i \omega_{n}\right)=\left\langle\left\langle\frac{1}{\beta} \sum_{i j} e^{i \omega_{n}\left(\tau_{i}-\tau_{j}\right)}\left(e^{V(i)}-1\right)\left[\mathbf{G G}_{0}^{-1}\right]_{i j}\right\rangle\right\rangle .
$$

Here the matrix $\mathbf{G}$ is calculated at each update of the auxiliary fields and $\mathbf{G}_{0}^{-1}$ is calculated and stored at the beginning of computation. The last term in Eq. (41) vanishes due to the time translational symmetry and

$$
\mathcal{G}\left(i \omega_{n}\right)=\mathcal{G}_{0}\left(i \omega_{n}\right)+\mathcal{G}_{0}\left(i \omega_{n}\right) \mathcal{S}\left(i \omega_{n}\right) \mathcal{G}_{0}\left(i \omega_{n}\right)
$$

As shown in Fig. 罒(c), the unphysical structure at $\omega_{n} \approx 0$ disappeared even at $\Delta \tau=1 / 5$ after the discretization errors have been corrected. Figure 5(d) at a finite bias $\Phi$ shows less curvature in the self-energy, which suggests that the correlation effects become weaker as bias increases. Green's functions evaluated this way showed excellent agreement with the continuous-time $\mathrm{QMC}^{34}$ at low fermion frequencies $i \omega_{n}$ at large Matsubara frequencies $i \varphi_{m}$ with computationally accessible $\Delta \tau$. In the calculations presented below used $\Delta \tau=1 / 5$ unless mentioned otherwise.

The self-energy of the QD Green's function $\Sigma\left(i \omega_{n}, i \varphi_{m}\right)$ is then computed in the same manner as in equilibrium theory, via the Dyson's equation

$$
\Sigma\left(i \omega_{n}, i \varphi_{m}\right)=\left[\mathcal{G}^{0}\left(i \omega_{n}\right)\right]^{-1}-\left[\mathcal{G}\left(i \omega_{n}\right)\right]^{-1}
$$

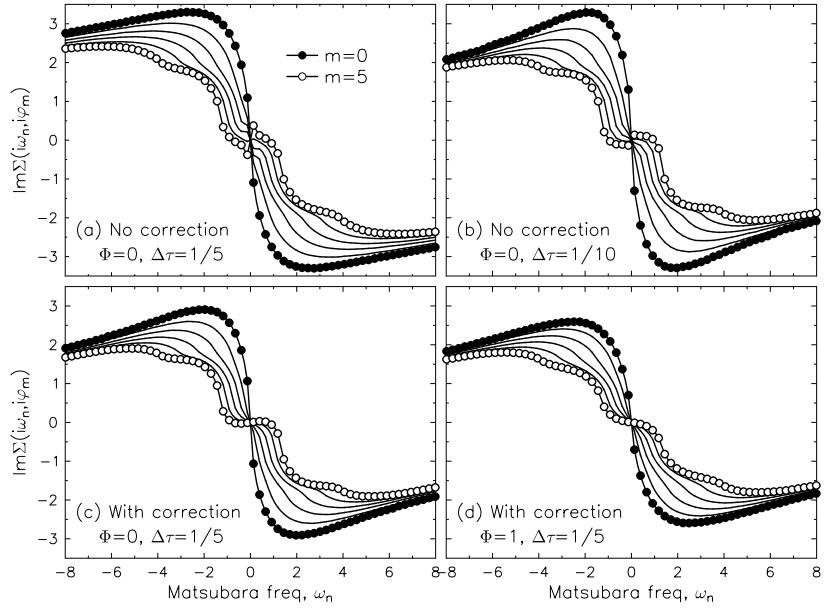

FIG. 5: Imaginary-time electron self-energy at $U=10$ and $\beta=24$ for Matsubara voltages $\varphi_{m}$ with $m=$ 0 (filled circle), $\cdots, 5$ (open circle). (a) Conventional HirschFye algorithm without the discretization correction shows spurious structures at small $\omega_{n}$ and finite $\varphi_{m}$. (b) With smaller discretization $\Delta \tau=1 / 10$, the spurious structures become weaker. (c) The discretization correction produced smooth self-energy at small $\omega_{n}$. (d) At higher bias $\Phi=1$, the curvature at high $\varphi_{m}$ becomes weaker, suggesting reduced correlation effects. The unit of energy is given by the noninteracting level width of $\mathrm{QD}, \Gamma=1$.

This self-energies $\Sigma\left(i \omega_{n}, i \varphi_{m}\right)$ at different $i \varphi_{m}$ values are computed in separate sets of QMC runs at each $i \varphi_{m}$. The numerical data for $\Sigma\left(i \omega_{n}, i \varphi_{m}\right)$ is analytically continued to the retarded self-energy $\Sigma^{r e t}(\omega)$ with the realfrequency $\omega$. The numerical procedure will be fully discussed in the next section.

We comment on why we choose to analytically continue the self-energy instead of the Green's function directly. As will be clear in subsequent discussions, the analytic form of the perturbative energy self-energy is more readily written down, which makes the numerical procedure more transparent. From the numerical standpoint of the discrete-time QMC, the discretization makes the high Matsubara frequency data less reliable for $\Sigma\left(i \omega_{n}, i \varphi_{m}\right)$ and $\mathcal{G}\left(i \omega_{n}, i \varphi_{m}\right)$. However, the systematic errors in an analytically continued $\Sigma(\omega)$ at large $\omega$ are less problematic since the frequency term $\omega$ in the Dyson's equation $G^{r e t}(\omega)=\left[\omega-\epsilon_{d}+i \Gamma-\Sigma(\omega)\right]^{-1}$ dominates $\Sigma(\omega)$.

\section{NUMERICAL ANALYTIC CONTINUATION}

\section{A. The spectral ansatz}

It seems a formidable task to perform an analytic continuation on numerical data in $\Sigma\left(i \omega_{n}, i \varphi_{m}\right)$. To guide the analytic continuation to a correct form we start with the self-energy in the second order of Coulomb interaction in the Anderson model, with the diagram depicted 


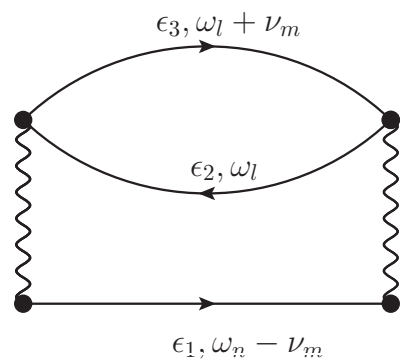

FIG. 6: Self-energy diagram of second order perturbation in the Coulomb parameter $U$ of the Anderson model.

in Fig. 6. The retarded self-energy can be easily calculated from $\Sigma \gtrless(t)=U^{2}\left[G_{0}^{\gtrless}(t)\right]^{2} G_{0}^{\lessgtr}(-t)$ and $\Sigma^{r e t}(t)=$ $\theta(t)\left[\Sigma^{>}(t)-\Sigma^{<}(t)\right]$ with the step-function $\theta(t)$,

$$
\begin{aligned}
\Sigma^{r e t}(\omega)= & U^{2} \sum_{\alpha_{1}, \alpha_{2}, \alpha_{3}}\left[\prod_{i=1}^{3} \frac{\Gamma_{i}}{\Gamma} \int d \epsilon_{i} \rho_{0}\left(\epsilon_{i}\right)\right] \\
& \times \frac{f_{1}\left(1-f_{2}\right) f_{3}+\left(1-f_{1}\right) f_{2}\left(1-f_{3}\right)}{\omega+i \eta-\epsilon_{1}+\epsilon_{2}-\epsilon_{3}},
\end{aligned}
$$

with the shorthand notation $f_{i}=f_{\alpha_{i}}\left(\epsilon_{i}\right) . \rho_{0}(\epsilon)$ is the non-interacting QD spectral function. If we do the same diagram in the imaginary-time formalism with the Hamiltonian $\hat{K}$, the self-energy is

$$
\begin{aligned}
\Sigma\left(i \omega_{n}, i \varphi_{m}\right)= & U^{2} \sum_{\alpha_{1}, \alpha_{2}, \alpha_{3}}\left[\prod_{i=1}^{3} \frac{\Gamma_{i}}{\Gamma} \int d \epsilon_{i} \rho_{0}\left(\epsilon_{i}\right)\right] \\
& \times \frac{f_{1}\left(1-f_{2}\right) f_{3}+\left(1-f_{1}\right) f_{2}\left(1-f_{3}\right)}{i \omega_{n}-\tilde{\epsilon}_{1}+\tilde{\epsilon}_{2}-\tilde{\epsilon}_{3}}
\end{aligned}
$$

with $\tilde{\epsilon}_{i}=\epsilon_{i}+\alpha_{i}\left(i \varphi_{m}-\Phi\right) / 2$. Here we have used the relation for the Fermi-Dirac function,

$$
f\left(\epsilon+\alpha \frac{i \varphi_{m}-\Phi}{2}\right)=f\left(\epsilon-\alpha \frac{\Phi}{2}\right)=f_{\alpha}(\epsilon),
$$

which is equivalent to Eq. (10). By combining the reservoirs indices

$$
\gamma=\alpha_{1}-\alpha_{2}+\alpha_{3}
$$

and the energy of an electron dressed by an electronhole pair as $\epsilon=\epsilon_{1}-\epsilon_{2}+\epsilon_{3}$, we can rewrite the above expression as

$$
\Sigma\left(i \omega_{n}, i \varphi_{m}\right)=\sum_{\gamma= \pm 1, \pm 3} \int d \epsilon \frac{\sigma_{\gamma}(\epsilon)}{i \omega_{n}-\frac{\gamma}{2}\left(i \varphi_{m}-\Phi\right)-\epsilon},
$$

with the spectral function $\sigma_{\gamma}(\epsilon)$ defined as

$$
\begin{aligned}
\sigma_{\gamma}(\epsilon)= & \pi U^{2} \sum_{\alpha_{1}, \alpha_{2}, \alpha_{3}}^{\alpha_{1}-\alpha_{2}+\alpha_{3}=\gamma}\left[\prod_{i=1}^{3} \frac{\Gamma_{i}}{\Gamma} \int d \epsilon_{i} \rho_{0}\left(\epsilon_{i}\right)\right] \\
& \times\left[f_{1}\left(1-f_{2}\right) f_{3}+\left(1-f_{1}\right) f_{2}\left(1-f_{3}\right)\right] \\
& \times \delta\left(\omega-\epsilon_{1}+\epsilon_{2}-\epsilon_{3}\right) .
\end{aligned}
$$

In the second order of interaction the self-energy spectral function $\sigma_{\gamma}(\epsilon)$ is independent of $i \varphi_{m}-\Phi$. However in the higher order of perturbation, it is no longer the case and we need to incorporate the $i \varphi_{m}-\Phi$ dependence in the spectral function as

$$
\Sigma\left(i \omega_{n}, i \varphi_{m}\right)=\sum_{\gamma=o d d Z} \int d \epsilon \frac{\sigma_{\gamma}(\epsilon) Q_{\gamma}\left(\epsilon, i \varphi_{m}-\Phi\right)}{i \omega_{n}-\frac{\gamma}{2}\left(i \varphi_{m}-\Phi\right)-\epsilon},
$$

with any odd integer $\gamma \cdot Q_{\gamma}\left(\epsilon, i \varphi_{m}-\Phi\right)$ is the correction due to higher order diagrams. See the appendix for detailed discussions for this generalization of the spectral representation and its analytic properties. We approximate the function $Q_{\gamma}$ by a Padé approximant

$$
Q_{\gamma}(\epsilon, z)=\frac{1+C_{\gamma}^{(1)}(\epsilon) z+C_{\gamma}^{(2)}(\epsilon) z^{2}+\cdots}{1+D_{\gamma}^{(1)}(\epsilon) z+D_{\gamma}^{(2)}(\epsilon) z^{2}+\cdots} .
$$

Therefore we seek the best spectral representation of the QMC-computed self-energy by treating $\left\{\sigma_{\gamma}(\epsilon), C_{\gamma}^{(n)}(\epsilon), D_{\gamma}^{(n)}(\epsilon)\right\}$ as fitting parameters. In the following calculations, we limit the $\gamma$-branches to $\gamma=$ $\pm 1, \pm 3, \pm 5, \pm 7$ and the Padé approximants to the first order $n=1$, which already required fitting 24 functions simultaneously. We will discuss in the next section the effects of the Padé approximants. We emphasize that, although the Padé coefficient functions $\left\{C_{\gamma}^{(n)}(\epsilon), D_{\gamma}^{(n)}(\epsilon)\right\}$ are adjusted in the numerical fit, they do not contribute to the (real-frequency) self-energy after the analytic continuation $i \varphi_{m} \rightarrow \Phi$

$$
\operatorname{Im} \Sigma^{r e t}(\omega)=-\pi \sum_{\gamma} \sigma_{\gamma}(\omega) .
$$

Real part of $\Sigma^{r e t}(\omega)$ is obtained from the Kramers-Kronig relation.

The electron self-energy satisfies the general relation

$$
\Sigma\left(i \omega_{n}, i \varphi_{m}\right)=\left[\Sigma\left(-i \omega_{n},-i \varphi_{m}\right)\right]^{*},
$$

as can be seen in the non-interacting Green's function, Eq. (32). For a particle-hole symmetric system, the noninteracting Green's function $\mathcal{G}_{0}\left(i \omega_{n}, i \varphi_{m}\right)$ in Eq. (32) is invariant with $i \varphi_{m}-\Phi \leftrightarrow-i \varphi_{m}+\Phi$ and we derive symmetry relations

$$
\begin{aligned}
\sigma_{\gamma}(\epsilon) & =\sigma_{-\gamma}(-\epsilon) \\
Q_{\gamma}\left(\epsilon, i \varphi_{m}-\Phi\right) & =Q_{-\gamma}\left(-\epsilon, i \varphi_{m}-\Phi\right) .
\end{aligned}
$$

In the previous work ${ }^{22}$, the relation $\sigma_{\gamma}(\epsilon)=\sigma_{-\gamma}(-\epsilon)$ has been incorrectly applied as $\sigma_{\gamma}(\epsilon)=\sigma_{-\gamma}(\epsilon)$ and this led to overly rapid reduction of the Kondo resonance at finite bias. As pointed out $\stackrel{23}{ }$ later, correct constraint produced a good agreement with other method ${ }^{9}$ in the moderately interacting limit, $U=5$ [see Fig. 2(d) in Ref ${ }^{23}$ ]. In this work, we do not impose any symmetry relations in the fit, and the resulting spectral functions recovered the above relations numerically. 


\section{B. Fitting procedures}

With the above spectral ansatz, we perform the leastsquare fit to the numerical self-energy generated by the QMC calculations with $\chi^{2}$ defined as

$$
\chi^{2}=\mathcal{N}^{-1} \sum_{n=-N}^{N-1} \sum_{m=0}^{M} \sigma_{n m}^{-2}\left|\frac{\Delta \Sigma\left(i \omega_{n}, i \varphi_{m}\right)}{\Sigma_{Q M C}\left(i \omega_{n}, i \varphi_{m}\right)}\right|^{2} .
$$

The deviation between the self-energy fit $\Sigma_{f i t}\left(i \omega_{n}, i \varphi_{m}\right)$ in the above ansatz and the QMC generated data $\Sigma_{Q M C}\left(i \omega_{n}, i \varphi_{m}\right)$ is $\Delta \Sigma\left(i \omega_{n}, i \varphi_{m}\right)=\Sigma_{f i t}\left(i \omega_{n}, i \varphi_{m}\right)-$ $\Sigma_{Q M C}\left(i \omega_{n}, i \varphi_{m}\right)$. We fit more accurately the low frequency self-energy data with an effective cutoff function

$$
\sigma_{n m}^{-2}=\frac{\Gamma^{2}}{\omega_{n}^{2}+\Gamma^{2}} \frac{\Gamma^{2}}{\varphi_{m}^{2}+\Gamma^{2}} .
$$

The normalization factor $\mathcal{N}$ is defined as

$$
\mathcal{N}=\sum_{n=-N}^{N-1} \sum_{m=0}^{M} \sigma_{n m}^{-2} .
$$

In the following calculations we used $M=5$ and $\omega_{N}=$ $8.0 \Gamma$.

In QMC applications, the analytic continuation has been one of the most controversial topics. Since the transformation of imaginary-time data to real-time information is an ill-defined procedure, noise in the imaginarytime data can lead to severe uncertainties in spectral functions. In the past, several algorithms have been proposed and the maximum entropy method based on the Bayesian inference $\frac{37}{3}$ and the method of stochastic image generation $^{38}$ have been widely used. In this work, we have not incorporated such methods where the focus so far has been limited to finding right spectral representations. Simultaneously finding a fit to many functions (24 as noted above) has already been quite an extensive task computationally. Refining the analytic continuation method remains an important area of future improvement for the imaginary-time theory of nonequilibrium.

We discretize the frequency on logarithmic mesh systems with 201 frequency points centered at $\omega=0$ over the energy range $[-30,30]$. Minimization of $\chi^{2}$ is achieved iteratively by using the Newton's steepest gradient method 39 . Once the fit reaches a certain threshold of accuracy $\left(\sqrt{\chi^{2}}<0.06\right)$, we regularized the spectral functions through third-order polynomial smoothing to reduced unwanted noise. The smoothing has had mostly insignificant effects and often has been unnecessary. The fractional error $\sqrt{\chi^{2}}$ in the fit resulted in the range of $1-6 \%$. Generally, the Padé approximant term produced better fits. Due to the dense frequency points near $\omega=0$, the update of spectral functions at small frequencies tends to be very slow. Therefore, for faster convergence, we used adjustable mesh systems which evolved from a coarse to a dense frequency mesh as the iterations progressed.

\section{Calculation of conductance}

Once the retarded Green's function is obtained, the current in a single-quantum model can be computed from the Meir-Wingreen's formula ${ }^{31}$,

$$
I=\frac{e}{\hbar} \Gamma \int d \epsilon A(\epsilon, \Phi)\left[f_{L}(\epsilon)-f_{R}(\epsilon)\right],
$$

with the QD spectral function at the bias $\Phi$,

$$
A(\epsilon, \Phi)=-\frac{1}{\pi} \operatorname{Im} \frac{1}{\omega-\epsilon_{d}-\Sigma^{r e t}(\omega)} .
$$

The differential conductance $G$ is obtained from numerical differentiation of the current by

$$
G=e \frac{d I}{d \Phi} .
$$

The differentiation has to be taken on discrete values of $\Phi_{i}(i=0,1, \cdots)$. For $\Phi_{i}=0$, the linear conductance is obtained from

$$
G(\Phi=0) / G_{0}=\pi \Gamma \int d \epsilon A(\epsilon, 0)\left(-\frac{\partial f(\epsilon)}{\partial \epsilon}\right),
$$

with the conductance quantum $G_{0}$ given by

$$
G_{0}=\frac{2 e^{2}}{h} .
$$

For the first non-zero bias $(i=1)$, we evaluate the derivatives from a third-order polynomial at $\Phi=\Phi_{1}$ determined from the values of current $\left\{-I_{2},-I_{1}, 0, I_{1}, I_{2}\right\}$ at bias $\left\{-\Phi_{2},-\Phi_{1}, 0, \Phi_{1}, \Phi_{2}\right\}$ as,

$$
G_{1}=\frac{1}{3} \frac{I_{1}+I_{2}}{\Delta \Phi}
$$

with $\Phi_{i}=i \Delta \Phi(i=0,1,2)$. For higher bias $\Phi_{i}(i>1)$,

$$
G_{i}=\frac{1}{2}\left[\frac{I_{i+1}-I_{i}}{\Phi_{i+1}-\Phi_{i}}+\frac{I_{i-1}-I_{i}}{\Phi_{i-1}-\Phi_{i}}\right] .
$$

\section{Comparison to other methods}

To demonstrate the validity of the imaginary-time QMC (ITQMC), the differential conductance ( $G=$ $d I / d V)$ is compared to other methods where data is available. Comparison to other methods of fRG $14,15,17$, ISPI 16.17, tDMRG ${ }^{11}$ is shown for the weakly interacting limit in Fig. Z(a). In such limit the spectral ansatz, Eq. (50), becomes an exact representation of the selfenergy at all bias and the resulting conductance is in good agreement with other methods. Even in the intermediate coupling limit $U / \Gamma=5$ in (b), the comparison to the time-dependent numerical renormalization group $\left(\mathrm{tNRG}^{9}\right)$ results is very good. Based on this concrete comparison and numerical efficiency of QMC for tackling complex QD models in strongly correlated regime, this imaginary-time method provides an efficient tool in nonequilibrium transport theory. 

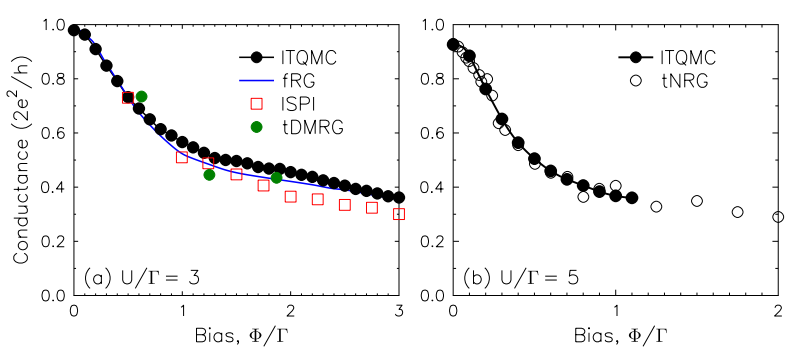

FIG. 7: (a) Comparison of differential conductance in weakly interacting limit at $U / \Gamma=3$ from the imaginary-time QMC (ITQMC) at $\beta=25$, functional renormalization group ( fRG $^{14,15,17}$, data taken from ${ }^{17}$ ), iterative summation of path integral (ISPI ${ }^{16,17}$ ), time-dependent density matrix renormalization group ( $\mathrm{DMMR}^{11}$ ). (b) Conductance in the intermediate coupling regime at $U / \Gamma=5$. Curves are from ITQMC $(\beta=24)$ and the time-dependent numerical renormalization group (tNRG ${ }^{9}$ at $\beta=25$ ).

\section{RESULTS AND DISCUSSIONS}

The spectral functions of the self-energy from the ansatz Eqs. (52 53) are shown in Fig. 8 and they demonstrate the nature of quasi-particles dressed with particlehole pairs. The parameters are $U=10, \beta=36$ and $\Phi=0.5$. In addition to the Kondo resonance at zero frequency, there are structures in the spectral functions at $\omega=\Phi / 2$ for $\gamma=1, \omega=3 \Phi / 2$ for $\gamma=3$, etc. For $\gamma=1$, the sum of reservoir indices of a dressed electron has $\sum_{i} \frac{\alpha_{i}}{2}=\frac{1}{2}$ and this leads to a resonant structure at the electron frequency measured from the combined chemical potential $\frac{1}{2} \Phi$. Similarly, there is a resonant structure at $\omega=\frac{3}{2} \Phi$ for $\gamma=3$. These effects of cross-lead particle-hole excitations appear as shoulders in the spectral function $A(\omega)$ in Fig. 9. The magnitude of the Padé terms $C_{\gamma}^{(1)}(\omega)$ and $D_{\gamma}^{(1)}(\omega)$ is much smaller than one, and this suggests that the higher-order Padé approximants will not significantly change the data presented here. We also note that the symmetry relations Eq. (56) have been numerically verified.

Spectral functions in the strongly correlated limit $U=$ 10 and 14 are shown in Fig. 9. In (a), the Kondo resonance develops sharply on top of the incoherent charge excitations with the Hubbard peaks at $\omega \sim \pm U / 2$. The inset shows the spectral function for the whole frequency range. The bias values are $\Phi=0,0.2,0.6,1.0,1.5$ and 2.0 (top to bottom curves). For clarity the curves are shifted vertically by 0.05 . The Kondo resonance is strongly quenched as the bias is applied. There appear spectral shoulders at $\omega=\Phi / 2$ (single vertical lines) and at $\omega=3 \Phi / 2$ (double vertical lines). Their strength is considerably weaker than reported in other works ${ }^{9,42}$.

The Kondo temperature is estimated from the halfwidth-at-half-maximum (HWHM) of the Kondo peak at zero bias $\Phi=0$. Due to the incoherent charge background at $\pi \Gamma A(\omega) \approx 0.2$, we read off the HWHM at

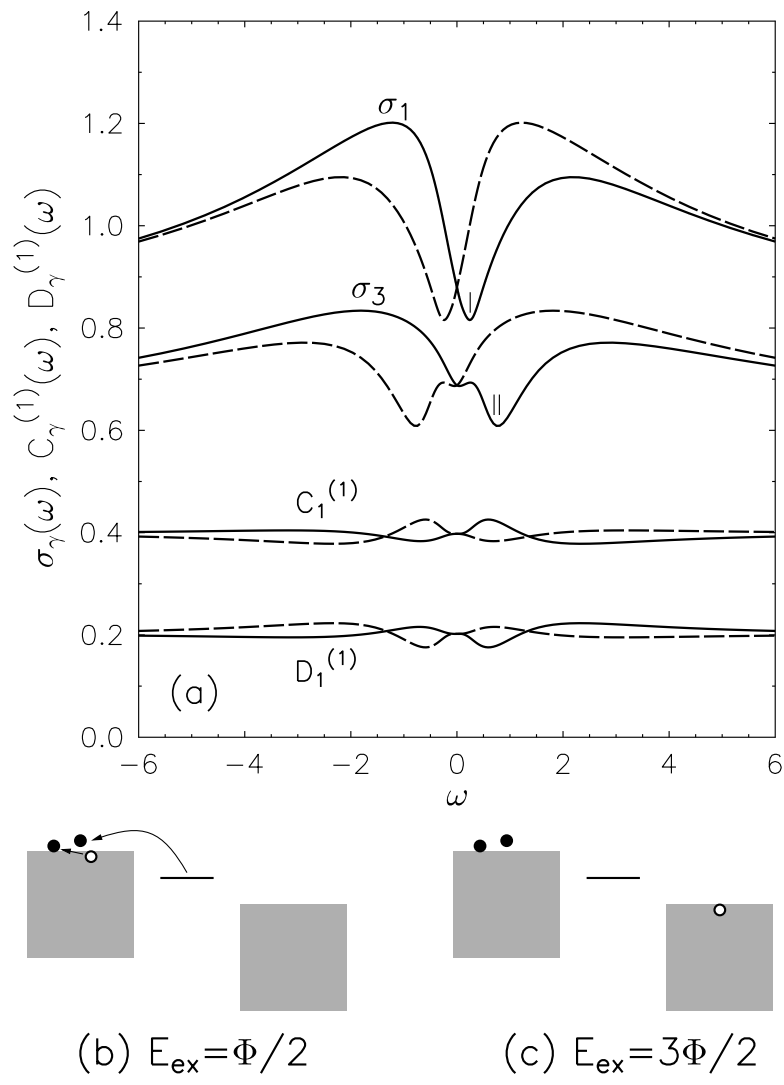

FIG. 8: (a) Spectral functions for the ansatz Eqs. (52, 53) at $U=10, \beta=36$ and $\Phi=0.5$. Dashed lines are for negative $\gamma^{\prime}$, eg. $\sigma_{-1}(\omega), \sigma_{-3}(\omega)$, etc. As expected, $\sigma_{\gamma}(\epsilon)=\sigma_{-\gamma}(-\epsilon)$ is satisfied in the particle-hole symmetric limit. Short vertical lines indicate $\omega=\Phi / 2$ (single) and $\omega=3 \Phi / 2$ (double line). (b) Intra-lead single-particle excitations dressed by particlehole excitations. They contribute to $\sigma_{1}(\omega)$ at excitation energy of $\Phi / 2$. (c) Inter-lead excitations for $\sigma_{3}(\omega)$ at excitation energy of $3 \Phi / 2$.

$\pi \Gamma A\left(\omega_{K}\right)=0.6$ and $\omega_{K}=0.075$. The Kondo temperature from the renormalization group $(R G)$ theory in the strong coupling regime $\frac{40,41}{4}$ has

$$
T_{K}^{R G}=\sqrt{\frac{U \Gamma}{2}} \exp \left(-\frac{\pi U}{8 \Gamma}+\frac{\pi \Gamma}{2 U}\right),
$$

with the HWHM $\omega_{K}^{R G}$ at

$$
\omega_{K}^{R G}=\frac{4}{\pi} T_{K}^{R G}=0.066,
$$

in a reasonable agreement with our numerical estimate.

Nonlinear conductance for $U=10$ is shown in Fig. 10 (a) with Padé approximants to the first order and (b) without the Padé correction. The comparison demonstrates that the corrections are insignificant, at least in the particle-hole symmetric Anderson model. Since the current is an integral of the spectral function, details in the spectral weight shift tend to be insensitive to different approximations of analytic continuation. The broken 

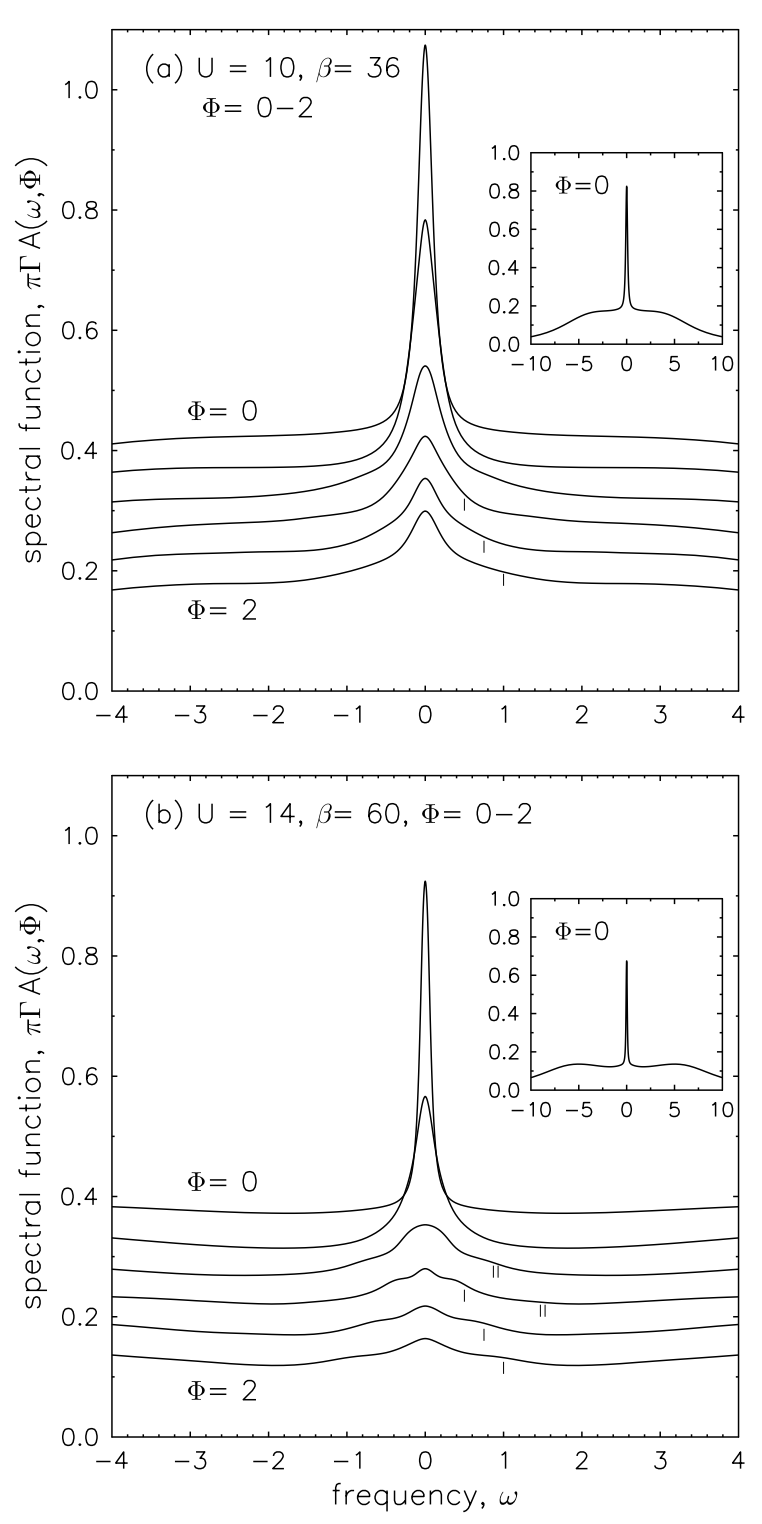

FIG. 9: (a) Spectral functions of one-particle Green's function at $U=10, \beta=36$ and bias voltage $\Phi=0,0.2,0.6,1.0,1.6,2.0$. The Kondo resonance becomes quenched quickly as the bias $\Phi$ is applied. The spectral functions are shifted by 0.05 for clarity. Short vertical lines mark spectral features at $\omega=\Phi / 2$. Inset: spectral function for the whole frequency range. (b) Spectral functions at $U=14, \beta=48$. Double vertical lines denote the spectral contributions at $\omega=3 \Phi / 2$. At larger $U$ the Kondo peaks get quenched faster to lower intensity.

lines in (a) are derived from Eq. (60) with the equilibrium spectral function $A_{e q}(\omega)$ calculated at $\Phi=0$. Therefore, the reduced ZBA width (about $50-60 \%$ ) in the full nonequilibrium calculations are due to the destruction of the Kondo resonance at finite bias.

For a comparison to experiments and other theories, we estimate first the temperature $T_{1 / 2}$ at zero bias at which the linear conductance becomes the half conductance quantum, $G\left(T_{1 / 2}, \Phi=0\right)=\frac{1}{2} G_{0} . T_{1 / 2} \approx 1 / 14=0.071$ at a similar energy scale with the above HWHM $\omega_{K}=$ 0.075 and $\omega_{K}^{R G}=0.066$. Then, from Fig. 9(a), we estimate the bias for half conductance quantum at the minimum temperature of simulation $T_{\min }, G\left(T_{\min }, \Phi_{1 / 2}\right)=$ $\frac{1}{2} G_{0}$ is estimated to be $\Phi_{1 / 2} \approx 0.135$ at $T_{\min }=1 / 60$. From the phenomenological scaling form ${ }^{3,41}$ of the conductance in the leading order of temperature and bias is written as

$$
\frac{G(T, \Phi)}{G_{0}}=1-c_{T}\left(\frac{T}{T_{1 / 2}}\right)^{2}-c_{V}\left(\frac{\Phi}{T_{1 / 2}}\right)^{2}+\cdots
$$

By definition, $c_{T}=1 / 2$ and $c_{V}$ can be derived by solving $G\left(T_{\min }=1 / 60, \Phi_{1 / 2}\right)=\frac{1}{2} G_{0}$. Then we have an estimate for the ratio of the coefficients $\alpha$ at $U=10$ as

$$
\alpha_{q m c} \equiv\left(\frac{c_{V}}{c_{T}}\right)_{q m c} \approx \frac{T_{1 / 2}^{2}-T_{\min }^{2}}{\left(\Phi_{1 / 2}\right)^{2}} \approx 0.26 .
$$

Similar calculations have been repeated for $U=12$ and 14 and the results are summarized in TABLE I. Due to the small Kondo temperatures at large $U$, the maximum linear conductance at zero bias reached short of the conductance quantum at $G / G_{0}=0.73$ for $U=12, \beta=60$ and $G / G_{0}=0.63$ for $U=14, \beta=60$. The QMC estimates for $\alpha_{q m c}$ are about $\alpha_{q m c} \sim 0.2$. We note that the incoherent spectral background in the Anderson model is at $0.1-0.2$ in Fig. 9 with the conductance also approaching $0.1-0.2 G_{0}$ at high bias in Fig. 10, as opposed to theoretical predictions from the Kondo model or renormalized resonant level model. Therefore the above estimates of $T_{1 / 2}$ and $\Phi_{1 / 2}$, hence $\alpha_{q m c}$, should be taken with some caution when compared to other theoretical models. We also note that the estimates of $\alpha_{q m c}$ have been derived from finite values of $T_{1 / 2}$ and $\Phi_{1 / 2}$, instead of taking the limit $T, \Phi \rightarrow 0^{3.4}$.

For large $U$, the QMC calculations tend to produce overestimated Kondo resonance HWHM, $\omega_{K}$, compared to $\omega_{K}^{R G}$. It seems that a factor for the discrepancy is due to the discretization error despite much improved algorithm. Part of the problem could be from the analytic continuation where very sharp spectral peaks are fit with overestimated width. However, it is not clear at the moment, given the above values for $\alpha_{q m c}$, how such discrepancy affects the scaling behaviors.

\begin{tabular}{cccc}
\hline \hline$U$ & $\beta_{1 / 2}$ & $\Phi_{1 / 2} / \beta_{\min }$ & $\alpha_{q m c}$ \\
\hline 10 & 14 & $0.135 / 60$ & 0.26 \\
12 & 21 & $0.091 / 60$ & 0.24 \\
14 & 36 & $0.048 / 60$ & 0.21 \\
\hline \hline
\end{tabular}

TABLE I: Inverse temperature $\beta_{1 / 2}=1 / T_{1 / 2}$ for $G\left(T_{1 / 2}, \Phi=\right.$ $0)=\frac{1}{2} G_{0}$, bias $\Phi_{1 / 2}$ for $G\left(T_{\min }, \Phi_{1 / 2}\right)=\frac{1}{2} G_{0}$ at the minimum temperature of $T_{\min }=1 / \beta_{\min }$, and the scaling coefficients $\alpha_{q m c}$ derived for $U=10,12,14$. 

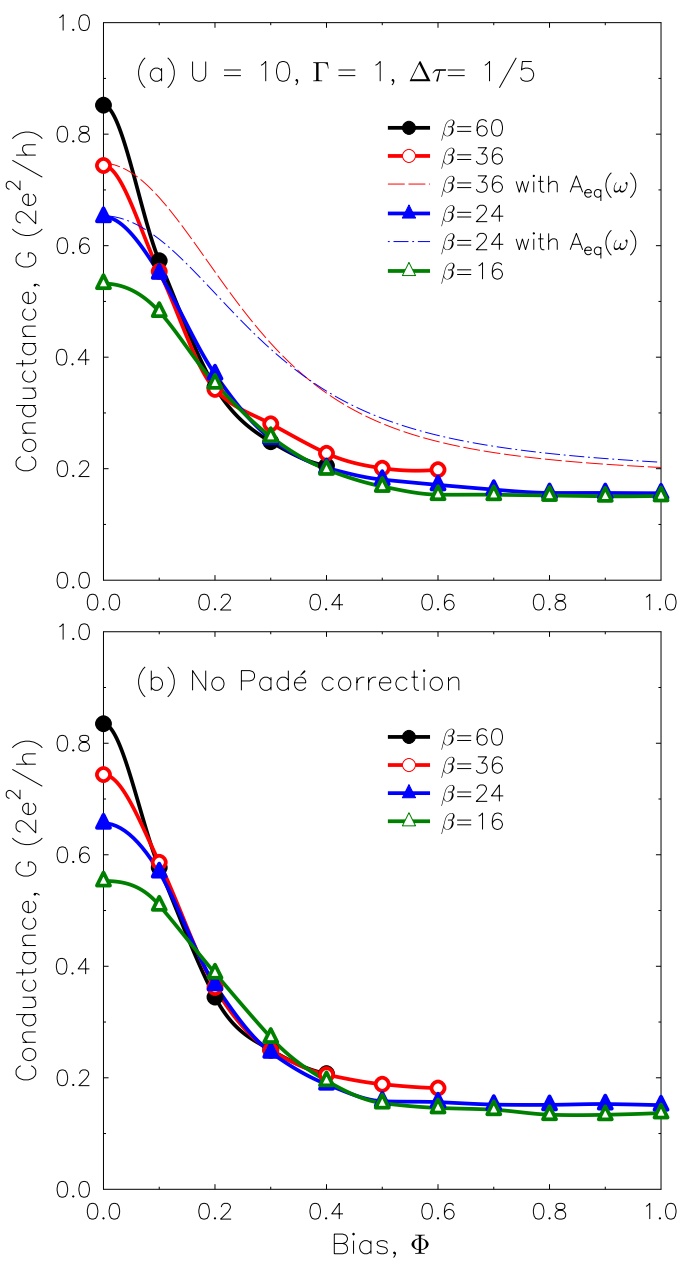

FIG. 10: (Color online) Conductance for $U=10$ at inverse temperatures $\beta=16,24,36$ and 60 . (a) Conductance curves calculated using equilibrium spectral function are shown with dashed lines. The narrower zero-bias anomaly width (about $50-60 \%$ from the equilibrium Kondo scale) indicates reduction of the Kondo effect at finite bias. (b) Conductance without the Padé correction. The difference is insignificant.

In a non-interacting resonant model with a rigid spectral function independent of temperature and bias, $\alpha_{0}=$ 0.25 can be easily obtained. Small $\alpha$ values can be interpreted as strong temperature dephasing of the Kondo resonance compared to that from bias voltage. Our ratios $\alpha_{q m c}$ have larger values than the perturbative estimate ${ }^{41} \alpha_{\text {pert }}=0.15$ from the effective Fermi liquid expansion. $\alpha_{q m c}$ falls within the experimental estimates which vary over a range of values, $\alpha_{\text {exp }}=0.05^{4}, 0.10^{3}$, $0.25^{2}$. We note that the conductance peak dies away at bias much smaller than where the spectral shoulders appear in Fig. 9. Therefore, such fine structures should not affect the scaling behavior. In general, the conductance results are much more robust than spectral function calculations.

\section{CONCLUSIONS}

Nonequilibrium imaginary-time theory has been formulated by introducing complex chemical potentials via the Matsubara voltage. It has been shown that the imaginary-time Green's functions upon analytic continuation are equivalent to the real-time Green's functions. For numerical analytic continuations we have given detailed discussions on the analytic structure of nonequilibrium spectral functions and generalized spectral representation in the strongly interacting regime. This formalism has an advantage of having familiar mathematical structure as in equilibrium theory and can be readily adopted for established equilibrium computational tools such as quantum Monte Carlo (QMC) method.

Application of the Hirsch-Fye QMC method to nonequilibrium has produced the nonlinear conductance physics where the Kondo resonance is strongly reduced by the external bias. The conductance peak has a reduced width from the prediction of equilibrium calculations. By correcting discretization errors in QMC, reliable conductance has been obtained as a function of temperature and bias. Using a scaling form of the conductance, we obtained coefficients to the leading temperature and bias dependent terms and their ratio $\alpha_{q m c} \sim 0.2$, larger than the perturbative prediction $\alpha_{\text {pert }}=0.15$ but within the experimental values. This suggests that nonperturbative effects lead to more rapid quenching of Kondo resonance at finite bias.

This work shows that the imaginary-time theory provides an effective computational tool, along with other numerical methods, in the fast-evolving field of nonequilibrium quantum many-body theory. This method has been applied to complex molecular quantum dot systems $^{23}$ and can be readily extended to bulk nonequilibrium using the dynamical mean-field theory, and quantum dot systems of complex geometry.

\section{ACKNOWLEDGEMENTS}

The author thanks Ryan Heary, Karyn Le Hur, Frithjof Anders, Akira Oguri, Reinhold Egger and David Goldhaber-Gordon for helpful discussions. Special thanks go to Andreas Dirks and Thomas Pruschke who pointed out the discretization problems in QMC and made their continuous-time QMC calculations available. Author is grateful for financial support from the National Science Foundation with the grant numbers DMR-0426826, DMR-0907150 and computing resources at CCR of SUNY Buffalo. 
(a)

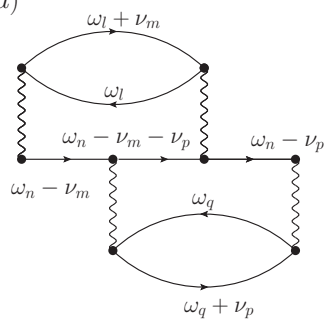

(c)

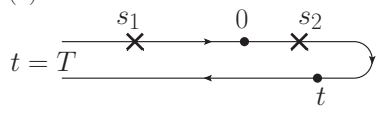

(b)

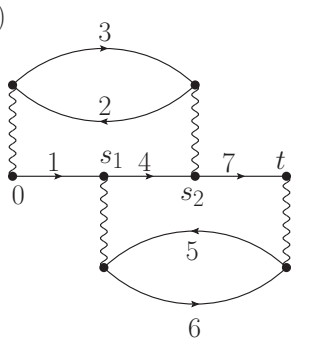

(d)

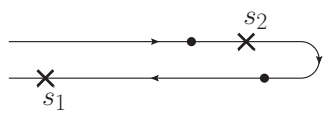

FIG. 11: (a) Fourth order vertex correction to the self-energy with the imaginary-frequency labels. (b) The same diagram in the real-time theory. Numerical labels denote scattering states, i.e. $1 \equiv\left(\alpha_{1}, k_{1}, \sigma_{1}\right)$. (c) Time-ordering for the greater Green's function with the interaction times $\left(s_{1}, s_{2}\right)$ with $s_{1}$ extending to $t=T$ on the upper Keldysh branch. (d) The same diagram with $s_{1}$ on the lower branch.

\section{Appendix A: Vertex correction and branch cuts for $z_{\varphi}=i \varphi_{m}-\Phi$ variable}

To examine the general analytic structure of the spectral representation of the imaginary-time self-energy, we go beyond the second-order contribution. Here we discuss that the spectral representation is expressed as

$$
\Sigma\left(i \omega_{n}, i \varphi_{m}\right)=\sum_{\gamma} \int d \epsilon \frac{\sigma_{\gamma}\left(\epsilon, i \varphi_{m}-\Phi\right)}{i \omega_{n}-\frac{\gamma}{2}\left(i \varphi_{m}-\Phi\right)-\epsilon}
$$

and how the analytic continuation $i \varphi_{m} \rightarrow \Phi \pm i \eta$ is taken. Up to the second order, the spectral function $\sigma_{\gamma}\left(\epsilon, i \varphi_{m}-\Phi\right)$ does not have any dependence on $i \varphi_{m}-\Phi$. To see how $\sigma_{\gamma}\left(\epsilon, i \varphi_{m}-\Phi\right)$ acquires the $i \varphi_{m}-\Phi$ dependence in the high order perturbation, we examine the vertex correction shown in Fig. 11(a). First, we express the polarization diagram $P_{0}\left(i \nu_{m}\right)$ as

$$
\begin{aligned}
P_{0}\left(i \nu_{m}\right) & =\frac{1}{\beta} \sum_{\omega_{n}} G_{0}\left(i \omega_{n}+i \nu_{m}\right) G_{0}\left(i \omega_{n}\right) \\
& =\frac{1}{\pi^{2} \beta} \sum_{\omega_{n}} \sum_{\alpha_{1}, \alpha_{2}} \int d \epsilon_{1} \int d \epsilon_{2} \frac{\Gamma_{\alpha_{1}} \Gamma_{\alpha_{2}}\left|g\left(\epsilon_{1}\right)\right|^{2}\left|g\left(\epsilon_{2}\right)\right|^{2}}{\left(i \omega_{n}+i \nu_{m}-\frac{\alpha_{1}}{2}\left(i \varphi_{m}-\Phi\right)-\epsilon_{1}\right)\left(i \omega_{n}-\frac{\alpha_{2}}{2}\left(i \varphi_{m}-\Phi\right)-\epsilon_{2}\right)}
\end{aligned}
$$

Here we introduce short-hand notations, $\int_{i}=\sum_{\alpha_{i}} \int d \epsilon_{i}, \rho_{i}=\left(\Gamma_{i} / \pi\right)\left|g\left(\epsilon_{i}\right)\right|^{2}, \tilde{\epsilon}_{i}=\epsilon_{i}+\frac{\alpha_{i}}{2}\left(i \varphi_{m}-\Phi\right)$. Then

$$
P_{0}\left(i \nu_{m}\right)=\frac{1}{\beta} \sum_{\omega_{n}} \int_{1} \int_{2} \frac{\rho_{1} \rho_{2}}{\left(i \omega_{n}+i \nu_{m}-\tilde{\epsilon}_{1}\right)\left(i \omega_{n}-\tilde{\epsilon}_{2}\right)}=\int_{1} \int_{2} \frac{\rho_{1} \rho_{2}\left(f_{2}-f_{1}\right)}{i \nu_{m}-\tilde{\epsilon}_{1}+\tilde{\epsilon}_{2}}
$$

which can be rewritten as

$$
P_{0}\left(i \nu_{m}\right)=\sum_{\gamma=0, \pm 1} \int d \epsilon \frac{A_{\gamma}(\epsilon)}{i \nu_{m}-\gamma\left(i \varphi_{m}-\Phi\right)-\epsilon}
$$

with

$$
A_{\gamma}(\epsilon)=\int_{1} \int_{2} \rho_{1} \rho_{2}\left(f_{2}-f_{1}\right) \delta\left(\epsilon-\epsilon_{1}+\epsilon_{2}\right) \delta_{\gamma, \gamma_{1}-\gamma_{2}}
$$

The diagram in Fig. 11(a) becomes

$$
\Sigma_{(a)}\left(i \omega_{n}\right)=\frac{1}{\beta^{2}} \sum_{\nu_{m}, \nu_{p}} G_{0}\left(i \omega_{n}-i \nu_{m}\right) G_{0}\left(i \omega_{n}-i \nu_{m}-i \nu_{p}\right) P_{0}\left(i \nu_{m}\right) G_{0}\left(i \omega_{n}-i \nu_{p}\right) P_{0}\left(i \nu_{p}\right) .
$$


Summing over $i \nu_{m}$ gives the partial factor

$$
\int_{1} \int_{2} \int_{3} \rho_{1} \rho_{2} A_{3}\left[\frac{f_{1}-f_{2}}{\left(i \nu_{p}+\tilde{\epsilon}_{2}-\tilde{\epsilon}_{1}\right)\left(i \omega_{n}-i \nu_{p}-\tilde{\epsilon}_{3}-\tilde{\epsilon}_{2}\right)}-\frac{f_{1}+n_{3}}{\left(i \omega_{n}-\tilde{\epsilon}_{3}-\tilde{\epsilon}_{1}\right)\left(i \omega_{n}-i \nu_{p}-\tilde{\epsilon}_{3}-\tilde{\epsilon}_{2}\right)}\right],
$$

with the Bose-Einstein function $n_{i}=\left(e^{\beta \tilde{\epsilon}_{i}}-1\right)^{-1}=\left[e^{\beta\left(\epsilon_{i}-\alpha_{i} \Phi\right)}-1\right]^{-1}$. Performing the summation on $i \nu_{p}$ on the first term proportional to $f_{1}-f_{2}$ with the remaining factors in Eq. (A7), we have

$$
\begin{aligned}
& \frac{1}{\beta} \sum_{\nu_{p}} \frac{G_{0}\left(i \omega_{n}-i \nu_{p}\right) P_{0}\left(i \nu_{p}\right)}{\left(i \nu_{p}+\tilde{\epsilon}_{2}-\tilde{\epsilon}_{1}\right)\left(i \omega_{n}-i \nu_{p}-\tilde{\epsilon}_{3}-\tilde{\epsilon}_{2}\right)} \\
= & \int_{4} \int_{5} \frac{\rho_{4} A_{5}}{\tilde{\epsilon}_{4}-\tilde{\epsilon}_{2}-\tilde{\epsilon}_{3}}\left[\frac{n_{5}-n_{1-2}}{\tilde{\epsilon}_{5}+\tilde{\epsilon}_{2}-\tilde{\epsilon}_{1}}\left(\frac{1}{i \omega_{n}-\tilde{\epsilon}_{1}-\tilde{\epsilon}_{3}}-\frac{1}{i \omega_{n}-\tilde{\epsilon}_{1}+\tilde{\epsilon}_{2}-\tilde{\epsilon}_{4}}\right)\right. \\
& \left.+\frac{n_{5}+\bar{f}_{2+3}}{\left(i \omega_{n}-\tilde{\epsilon}_{2}-\tilde{\epsilon}_{3}-\tilde{\epsilon}_{5}\right)\left(i \omega_{n}-\tilde{\epsilon}_{1}-\tilde{\epsilon}_{3}\right)}-\frac{n_{5}+\bar{f}_{4}}{\left(i \omega_{n}-\tilde{\epsilon}_{4}-\tilde{\epsilon}_{5}\right)\left(i \omega_{n}-\tilde{\epsilon}_{1}+\tilde{\epsilon}_{2}-\tilde{\epsilon}_{4}\right)}\right],
\end{aligned}
$$

with $\bar{f}_{i}=1-f_{i}=\left(e^{-\beta \tilde{\epsilon}_{i}}+1\right)^{-1}=\left[e^{-\beta\left(\epsilon_{i}-\alpha_{i} \Phi / 2\right)}+1\right]^{-1}$. This expression can be reduced to the form

$$
\sum_{\gamma} \int d \epsilon \frac{B_{\gamma}\left(\epsilon, i \varphi_{m}-\Phi\right)}{i \omega_{n}-\frac{\gamma}{2}\left(i \varphi_{m}-\Phi\right)-\epsilon}
$$

by repeatedly using

$$
\frac{1}{i \omega_{n}-z_{1}} \frac{1}{i \omega_{n}-z_{2}}=\frac{1}{z_{1}-z_{2}}\left[\frac{1}{i \omega_{n}-z_{1}}-\frac{1}{i \omega_{n}-z_{2}}\right]
$$

It can be shown that the above form can be deduced for other types of high order perturbation diagrams. The form Eq. (A11) could have been anticipated from the Hamiltonian Eq. (11) where $i \varphi_{m}-\Phi$ serves as a parameter and the equilibrium imaginary-time theory has a similar spectral representation for electron self-energy due to the causality.

However there remains an important question concerning the direction of analytic continuation of $i \varphi_{m} \rightarrow \Phi$. The denominator $\left(i \omega_{n}-\frac{\gamma}{2}\left(i \varphi_{m}-\Phi\right)-\epsilon\right)^{-1}$ in Eq. (A11) does not pose a problem regarding the direction $i \varphi_{m} \rightarrow \Phi+i 0^{+}$ or $i \varphi_{m} \rightarrow \Phi-i 0^{+}$due to the finite imaginary number in $i \omega_{n}$. However, the factor $B_{\gamma}\left(\epsilon, i \varphi_{m}-\Phi\right)$ contains energy denominators such as $\left(\tilde{\epsilon}_{4}-\tilde{\epsilon}_{2}-\tilde{\epsilon}_{3}\right)^{-1}$ in Eq. (A10), which may result differently depending on the direction of the continuation $i \varphi_{m} \rightarrow \Phi \pm i 0^{+}$.

To resolve this issue, we examine how such energy denominators behave in the Keldysh real-time theory. We consider the same fourth order diagram as shown in Fig. 11(b). For a specific time ordering of Fig. 11(c) for $t>0$, its partial contribution to the self-energy $\Sigma^{>}(t)$ can be expressed as

$$
\begin{aligned}
& \int_{T}^{0} d s_{1} \int_{0}^{t} d s_{2}\left\langle d(t) d^{\dagger}\left(s_{2}\right)\right\rangle\left\langle d\left(s_{2}\right) d^{\dagger}\left(s_{1}\right)\right\rangle\left\langle d^{\dagger}(0) d\left(s_{1}\right)\right\rangle\left\langle d\left(s_{2}\right) d^{\dagger}(0)\right\rangle\left\langle d^{\dagger}\left(s_{2}\right) d(0)\right\rangle\left\langle d^{\dagger}(t) d\left(s_{1}\right)\right\rangle\left\langle d(t) d^{\dagger}\left(s_{1}\right)\right\rangle \\
= & \int_{T}^{0} d s_{1} \int_{0}^{t} d s_{2}\left[\prod_{i=1,7} \int_{i} \rho_{i}\right] f_{1} f_{2} \bar{f}_{3} \bar{f}_{4} f_{5} \bar{f}_{6} \bar{f}_{7} e^{-i \epsilon_{1} s_{1}+i \epsilon_{4}\left(s_{1}-s_{2}\right)+i \epsilon_{7}\left(s_{2}-t\right)-i \epsilon_{3} s_{2}+i \epsilon_{2} s_{2}+i\left(\epsilon_{5}-\epsilon_{6}\right)\left(t-s_{1}\right)},
\end{aligned}
$$

with the continuum labels defined in Fig. 111(c). Here we take the limit $T \rightarrow-\infty$ as prescribed by Gell-mann and Goldberg ${ }^{30}$ by taking the $T$-integral $\eta \int_{-\infty}^{0} d T e^{\eta T}$. Also, performing the integrals on $s_{1}$ and $s_{2}$ we get

$$
\left[\prod_{i=1,7} \int_{i} \rho_{i}\right] f_{1} f_{2} \bar{f}_{3} \bar{f}_{4} f_{5} \bar{f}_{6} \bar{f}_{7} \frac{e^{-i t\left(\epsilon_{3}-\epsilon_{2}+\epsilon_{4}-\epsilon_{5}+\epsilon_{6}\right)}-e^{-i t\left(\epsilon_{6}+\epsilon_{7}-\epsilon_{5}\right)}}{\left(\epsilon_{1}-\epsilon_{4}+\epsilon_{5}-\epsilon_{6}+i \eta\right)\left(\epsilon_{3}-\epsilon_{2}+\epsilon_{4}-\epsilon_{7}\right)} .
$$

Here, the bias dependence is only in the statistical factor $f_{1} f_{2} \cdots \bar{f}_{7}$. A different contribution to $\Sigma^{>}(t)$ is given in Fig. 11(d) with $s_{1}$ extending to $T$ on the lower Keldysh branch. Its contribution can be similarly calculated as

$$
-\left[\prod_{i=1,7} \int_{i} \rho_{i}\right] \bar{f}_{1} f_{2} \bar{f}_{3} f_{4} \bar{f}_{5} f_{6} \bar{f}_{7} \frac{e^{-i t\left(\epsilon_{3}-\epsilon_{2}+\epsilon_{4}-\epsilon_{5}+\epsilon_{6}\right)}-e^{-i t\left(\epsilon_{6}+\epsilon_{7}-\epsilon_{5}\right)}}{\left(\epsilon_{1}-\epsilon_{4}+\epsilon_{5}-\epsilon_{6}+i \eta\right)\left(\epsilon_{3}-\epsilon_{2}+\epsilon_{4}-\epsilon_{7}\right)},
$$


with the negative sign coming from different Wick contraction. The only difference from the previous expression is the statistical factor. Regarding the convergence factor $i \eta$, we are concerned with the contribution $A$

$$
A=\left[\prod_{i=1,4,5,6} \sum_{\alpha_{i}} \int d \epsilon_{i} \Gamma_{\alpha_{i}}\left|g_{i}\right|^{2}\right]\left(f_{1} \bar{f}_{4} f_{5} \bar{f}_{6}-\bar{f}_{1} f_{4} \bar{f}_{5} f_{6}\right) \frac{e^{-i t\left(\epsilon_{3}-\epsilon_{2}+\epsilon_{4}-\epsilon_{5}+\epsilon_{6}\right)}-e^{-i t\left(\epsilon_{6}+\epsilon_{7}-\epsilon_{5}\right)}}{\epsilon_{3}-\epsilon_{2}+\epsilon_{4}-\epsilon_{7}} \delta\left(\epsilon_{1}-\epsilon_{4}+\epsilon_{5}-\epsilon_{6}\right) .
$$

Within the constraint given by the $\delta$-function,

$$
f_{1} \bar{f}_{4} f_{5} \bar{f}_{6}-\bar{f}_{1} f_{4} \bar{f}_{5} f_{6}=f_{1} f_{4} f_{5} f_{6} e^{\beta\left(\epsilon_{4}+\epsilon_{6}\right)}\left[e^{-\beta\left(\alpha_{4}+\alpha_{6}\right) \Phi}-e^{-\beta\left(\alpha_{1}+\alpha_{5}\right) \Phi}\right] .
$$

In equilibrium $\Phi=0, A=0$ and the energy integral becomes principal value integral and the presence of $i \eta$ becomes irrelevant. The same holds in nonequilibrium for single quantum-dot systems by applying the same argument in Section [1] and Fig. 3. States (15) play the role of incoming state $|n\rangle$ and (46) the outgoing state $|m\rangle$ in Fig. 3(a). For example, for $\left(\alpha_{1} \alpha_{5}\right)=(L R)$ and $\left(\alpha_{4} \alpha_{6}\right)=(L L)$ there exists a permutation of reservoir labels in the $\alpha$-summation of Eq. (A17),$\left(\alpha_{1} \alpha_{5}\right)=(L L)$ and $\left(\alpha_{4} \alpha_{6}\right)=(L R)$ without permuting the energy variables $\left(\epsilon_{1}, \epsilon_{4}, \epsilon_{5}, \epsilon_{6}\right)$ and changing the factor $\Gamma_{1} \Gamma_{4} \Gamma_{5} \Gamma_{6}$. Therefore the expression $A$ becomes zero for nonequilibrium and the integrals of energy poles around the real axis due to $i \eta$ can be replaced by principal integrals. Finally the analytic continuation of $i \varphi_{m} \rightarrow \Phi$ can be taken as

$$
\left(i \varphi_{m} \rightarrow \Phi\right)=\frac{1}{2}\left[\left(i \varphi_{m} \rightarrow \Phi+i 0^{+}\right)+\left(i \varphi_{m} \rightarrow \Phi-i 0^{+}\right)\right]
$$

and the subtlety of the analytic continuation $\left(i \varphi_{m} \rightarrow \Phi\right)$ is resolved.

We write the total imaginary-time self-energy as

$$
\Sigma\left(i \omega_{n}, i \varphi_{m}\right)=\sum_{\gamma} \int d \epsilon \frac{\sigma_{\gamma}(\epsilon) Q_{\gamma}\left(\epsilon, i \varphi_{m}-\Phi\right)}{i \omega_{n}-\frac{\gamma}{2}\left(i \varphi_{m}-\Phi\right)-\epsilon}
$$

with the function $Q$ expressed as a Padé quotient

$$
Q_{\gamma}(\epsilon, z)=\frac{1+C_{\gamma}^{(1)}(\epsilon) z+C_{\gamma}^{(2)}(\epsilon) z^{2}+\cdots}{1+D_{\gamma}^{(1)}(\epsilon) z+D_{\gamma}^{(2)}(\epsilon) z^{2}+\cdots}
$$

In particle-hole asymmetric limit, one also needs to consider the constant term in addition to Eq. A20).

$$
\Sigma\left(i \omega_{n}, i \varphi_{m}\right)=\Sigma^{0}\left(i \varphi_{m}-\Phi\right)+\sum_{\gamma} \int d \epsilon \frac{\sigma_{\gamma}(\epsilon) Q_{\gamma}\left(\epsilon, i \varphi_{m}-\Phi\right)}{i \omega_{n}-\frac{\gamma}{2}\left(i \varphi_{m}-\Phi\right)-\epsilon} .
$$

$\Sigma^{0}\left(i \varphi_{m}-\Phi\right)$ is represented by another Padé approximant as

$$
\Sigma^{0}(z)=\Sigma^{0} \frac{1+c^{(1)} z+c^{(2)} z^{2}+\cdots}{1+d^{(1)} z+d^{(2)} z^{2}+\cdots} .
$$

In this work, we have only considered the particle-hole symmetric limit and the constant self-energy term $\Sigma^{0}\left(i \varphi_{m}-\Phi\right)$ has not been included in the analytic continuation.

1 S. M. Cronenwett, T. H. Oosterkamp, L. P. Kouwenhoven, Science 281, 540 (1998).

2 W. G. van der Wiel, S. De Franceschi, T. Fujisawa, J. M. Elzerman, S. Tarucha, and L. P. Kouwenhoven, Science 289, 2105 (2000).

3 M. Grobis, I. G. Rau, R. M. Potok, H. Shtrikman, and D. Goldhaber-Gordon, Phys. Rev. Lett. 100, 246601 (2008).
${ }^{4}$ G. D. Scott et al., Phys. Rev. B 79, 165413 (2009).

5 R. M. Potok, I. G. Rau, Hadas Shtrikman, Yuval Oreg and D. Goldhaber-Gordon, Nature 446, 167 (2007).

6 J. Rammer and H. Smith, Rev. Mod. Phys. 58, 323 (1986).

7 S. Datta, Electronic Transport in Mesoscopic Systems, Cambridge University Press, Cambridge UK (1995).

8 F. B. Anders and A. Schiller, Phys. Rev. Lett. 95, 196801 
(2005).

9 F. B. Anders, Phys. Rev. Lett. 101, 066804 (2008).

10 E. Boulat, H. Saleur, and P. Schmitteckert, Phys. Rev. Lett. 101, 140601 (2008).

11 F. Heidrich-Meisner, A. E. Feiguin, and E. Dagotto, Phys. Rev. B 79, 235336 (2009).

12 A. Rosch, J. Paaske, J. Kroha, and P. Wölfle, Phys. Rev. Lett. 90, 076804 (2003).

13 S. Kehrein, Phys. Rev. Lett. 95, 056602 (2005).

14 S. G. Jakobs, V. Meden and H. Schoeller, Phys. Rev. Lett. 99, 150603 (2007).

15 H. Schmidt, and P. Wölfle, Ann. Phys. (Berlin) 19, 60 (2010).

16 S. Weiss, J. Eckel, M. Thorwart, and R. Egger, Phys. Rev. B 77, 195316 (2008).

17 J. Eckel, F. Heidrich-Meisner, S.G. Jakobs, M. Thorwart, M. Pletyukhov, R. Egger, arXiv:1001.3773v1 (2010).

18 M. Schiro and Michele Fabrizio, Phys. Rev. B 79, 153302 (2009).

19 P. Werner, T. Oka, and A. J. Millis, Phys. Rev. B 79, 035320 (2009).

20 P. Mehta and N. Andrei, Phys. Rev. Lett. 96, 216802 (2006); Phys. Rev. Lett. 100, 086804 (2008).

21 B. Doyon, Phys. Rev. Lett. 99, 076806 (2007).

22 J. E. Han and R. J. Heary, Phys. Rev. Lett. 99, 236808 (2007).

23 J. E. Han, Phys. Rev. B 81, 113106 (2010).

24 R. M. Fye and J. E. Hirsch, Phys. Rev. B 38, 433 (1988).

25 D. N. Zubarev, Nonequilibrium Statistical Thermodynamics, Consultants Bureau, New York (1974).
26 S. Hershfield, Phys. Rev. Lett. 70, 2134 (1993).

27 Eugen Merzbacher, Quantum Mechanics, Chapter 21, John Wiley \& Sons, New York (1961).

28 J. E. Han, Phys. Rev. B 73, 125319 (2006).

29 J. E. Han, Phys. Rev. B 75, 125122 (2007).

30 M. Gell-Mann and M. L. Goldberger, Phys. Rev. 91, 398 (1953)

31 Y. Meir and N. S. Wingreen, Phys. Rev. Lett. 68, 2512 (1992).

32 R. Blankenbecler, D. J. Scalapino and R. L. Sugar, Phys. Rev. D 24, 2278 (1981).

33 J. W. Negele and H. Orland, Quantum many-particle systems, Addison-Wesley, USA (1988).

34 A. Dirks, P. Werner, M. Jarrell, and T. Pruschke, arXiv:1002.4081 (2010).

35 A. N. Rubtsov, V. V. Savkin, and A. I. Lichtenstein, Phys. Rev. B 72, 035122 (2005).

36 E. Gull, P. Werner, O. Parcollet and M. Troyer, arXiv:0802.3222 3 (2008).

37 Mark Jarrell and J. E. Gubernatis, Phys. Rep. 269, 133 (1996).

38 A. S. Mishchenko, N. V. Prokof'ev, A. Sakamoto, and B. V. Svistunov, Phys. Rev. B 62, 6317 (2000).

39 M. C. Payne, M. P. Teter, D. C. Allan, T. A. Arias and J. D. Joannopoulos, Rev. Mod. Phys. 64, 1045 (1992).

40 A. C. Hewson, The Kondo Problem to Heavy Fermions, Cambridge University Press, UK (1997).

41 A. Oguri, J. Phys. Soc. Jap. 74, 110 (2005).

42 T. Fujii and K. Ueda, Phys. Rev. B 68, 155310 (2003). 